Escuela DE Posgrado

Máster Oficial en Estructuras

Trabajo Fin de MÁster

\title{
Aplicación de Análisis Modal Operacional a Estructura Singular
}

Autor:

Eva María Ramos Paredes

Tutor:

Rafael Castro Triguero

Granada, 26 de Septiembre de 2012.

Curso académico 2011/12 



\section{Índice general}

\begin{tabular}{ll}
\hline Nomenclatura y Siglas. & 7
\end{tabular}

\begin{tabular}{lr}
\hline Introducción. & 9
\end{tabular}

Motivación y Objetivos . . . . . . . . . . . . . . . . . . . . . . 10

Estructura de la memoria . . . . . . . . . . . . . . . . . . . . . . 11

\begin{tabular}{ll}
\hline 1. Estado del Arte. & 13
\end{tabular}

1.1. Metodologías de localización de sensores. . . . . . . . . . . . . . . . . 13

1.2. Detección de daño estructural. . . . . . . . . . . . . . . . . . . . . . . 14

1.3. Actualización del modelo. . . . . . . . . . . . . . . . . . . . . . . . 15

1.4. Parámetros de incertidumbre. . . . . . . . . . . . . . . . . . . 15

\begin{tabular}{ll}
\hline 2. Modelo de Elementos Finitos. & 17
\end{tabular}

2.1. Precedentes. . . . . . . . . . . . . . . . . . . . 17

2.2. Descripción de la estructura. . . . . . . . . . . . . . . . . . . . . . 19

2.3. Cálculo en SAP2000. . . . . . . . . . . . . . . . . 23

2.4. Introducción de datos en Matlab. . . . . . . . . . . . . . . . . . . . . 24

3. Metodologías de localización de sensores. 27

3.1. Método de Independencia Efectiva (EFI). . . . . . . . . . . . . . . . . . . 27

3.2. Optimización del rango de la matriz de energía (EMRO). . . . . . . . . . . 29 
\begin{tabular}{lll}
\hline 4. Resultados. & 31
\end{tabular}

4.1. Cálculo determinista de las OSP. . . . . . . . . . . . . . . . . . . . . 31

4.1.1. Búsqueda de DOFs $\ldots \ldots \ldots \ldots \ldots \ldots$

4.1.2. Criterios de evaluación de metodologías OSP . . . . . . . . . . . . 34

4.2. Simulación de Monte Carlo (MCS). . . . . . . . . . . . . . . . . . . . . . 37

4.3. Localización de sensores bajo incertidumbre paramétrica . . . . . . . . . . 38

4.3.1. Efectos de las incertidumbres sobre la OSP . . . . . . . . . . . . 38

4.3.2. Criterios de evaluación de metodologías OSP . . . . . . . . . . . . 39

4.4. Configuración óptima de sensores con ruido. . . . . . . . . . . . . . . . . . 41

5. Conclusiones y Trabajo Futuro. $\quad 45$

5.1. Conclusiones. . . . . . . . . . . . . . . . . . . . . . 45

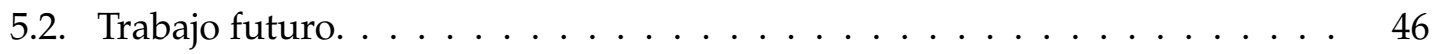

\begin{tabular}{lr}
\hline Bibliografía & 47
\end{tabular} 


\section{Índice de figuras}

2.1. Almacén de Herdez, Mexico D.F., 1955-1956. Félix Candela. . . . . . . . . . . . . . . . 18

2.2. Capilla de Nuestra Señora de la Soledad, Mexico D.F., 1956. Félix Candela. . . . . . . . 18

2.3. Propuesta para cubrir recinto polideportivo de $46 \times 24 \mathrm{~m} . \ldots \ldots$

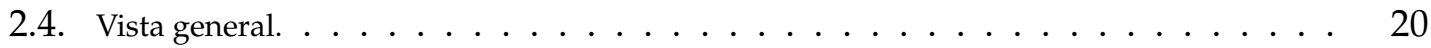

2.5. Vista lateral con cerramiento retráctil. . . . . . . . . . . . . . . . . . . 20

2.6. Detalle de soporte lateral. . . . . . . . . . . . . . . . . . . . . . . . . 21

2.7. Detalle de encuentro entre la lona y el marco estructural. . . . . . . . . . . . . . . . 22

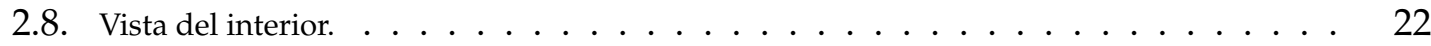

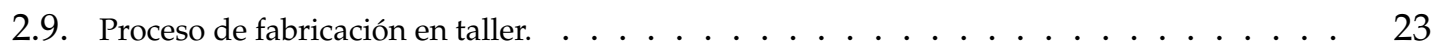

2.10. Transporte de soportes. . . . . . . . . . . . . . . . 23

2.11. Estructura modelizada. . . . . . . . . . . . . . . . . . . 24

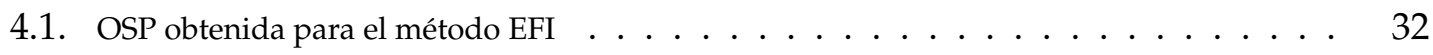

4.2. OSP obtenida para el método EFIWM . . . . . . . . . . . . . 33

4.3. OSP obtenida para el método KEMRO . . . . . . . . . . . . . . . . . . . . . . 33

4.4. OSP obtenida para el método SEMRO . . . . . . . . . . . . . . . . . . . . . . 34

4.5. Distintas soluciones tomando distintas incertidumbres $\ldots \ldots \ldots \ldots$

4.6. OSP obtenida para el método EFI . . . . . . . . . . . . . . . . . 39

4.7. Histogramas de los criterios de evaluación. . . . . . . . . . . . . . . . . . . 40

4.8. Histogramas del criterio de evaluación aplicando el MAC rms error para distintos niveles de ruido con 11 sensores. . . . . . . . . . . . . . . . . 43 


\title{
Nomenclatura y Siglas.
}

\author{
$\theta \mathrm{x} \quad$ Grado de libertad rotacional en $\mathrm{x}$ \\ ux Grado de libertad translacional en $x$ \\ $\varnothing \quad$ Diámetro \\ $\mathbf{m}^{2} \quad$ Metros cuadrados \\ g Gramos \\ $\rho \quad$ Densidad del elemento \\ E Módulo de Young del elemento \\ M Matriz de masa del elemento \\ K Matriz de rigidez del elemento \\ $\phi \quad$ Matriz de formas modales del elemento \\ GPa GigaPascales
}




\begin{tabular}{|c|c|c|}
\hline DOFs & Degrees of Freedom & Grados de libertad \\
\hline EFI & Efficience Independence Method & Método de Independencia efectiva \\
\hline EMA & Experimental Modal Analysis & Análisis Modal Experimental \\
\hline EMRO & Energy Matrix Rank Optimization & $\begin{array}{l}\text { Optimización del rango de la Matriz } \\
\text { de Energía }\end{array}$ \\
\hline FIM & Fisher Information Matrix & Matriz de Información de Fisher \\
\hline KEMRO & $\begin{array}{l}\text { Kinetic Energy Matrix Rank } \\
\text { Optimization }\end{array}$ & $\begin{array}{l}\text { Optimización del rango de la Matriz } \\
\text { de Energía Cinética }\end{array}$ \\
\hline MAC & Modal Assurance Criterion & Criterio de Aseguramiento Modal \\
\hline MCS & Monte Carlo Simulation & Simulación de Monte Carlo \\
\hline MDOFs & Multiple Degrees of Freedom & Grados de libertad múltiples \\
\hline OMA & Operational Modal Analysis & Análisis Modal Operacional \\
\hline OSP & Optimal Sensor Placement & Localización Óptima de Sensores \\
\hline SEMRO & $\begin{array}{l}\text { Strain Energy Matrix Rank } \\
\text { Optimization }\end{array}$ & $\begin{array}{l}\text { Optimización del rango de la Matriz } \\
\text { de Energía de Deformación }\end{array}$ \\
\hline SHM & Structural Health Monitoring & Monitorización de Condición Estructural \\
\hline SNR & Signal-to-noise ratio & Relación señal/ruido \\
\hline
\end{tabular}




\section{Introducción.}

El presente trabajo realiza un estudio de localización óptima de sensores de una estructura metálica con una geometría muy particular. Han sido aplicadas cuatro metodologías clásicas de localización de sensores: dos basados en la Matriz de Información de Fisher (en inglés Fisher Information Matrix, FIM) y otros basados en la optimización del rango de la matriz de energía. Al ser una estructura calculada y construida, tenemos un módulo de Young una densidad y un dimensionamiento de las secciones tubulares. Este estudio plantea introducir incertidumbres a esos datos ya fijos, para ver la influencia que tienen los mismos sobre el análisis modal obtenido. Además a lo ya existente se incorporará al modelo de elementos finitos los efectos de distintos niveles de ruido numéricamente para simular sus consecuencias. Se podrá observar que la influencia de incertidumbres paramétricas en la metodología de localización óptima de sensores puede ser muy significante a la hora de realizar una configuración de sensores óptima.

El emplazamiento de sensores en una estructura es un paso inicial importante en el campo del Análisis Modal Experimental (Experimental Modal Analysis, EMA) y en particular del Análisis Modal Operacional (Operational Modal Analysis, OMA). En el EMA los parámetros modales se obtienen de las funciones de respuesta en frecuencia que relacionan la salida (respuesta) con la entrada (excitación). Por otro lado, el OMA se basa en la localización de sensores que reciban la respuesta de la estructura usando sólo el ambiente y las fuerzas de servicio que actúan sobre la misma.

Además de definir la principal diferencia entre los dos sistemas, se procede a definir otras características. El OMA se emplea cuando el objeto de estudio no es conveniente excitarlo, ya sea por la complejidad de la estructura, dificultad de lectura o tamaño. Al analizar la respuesta sin una excitación programada, debemos contar con unos equipos de adquisición muy sensibles ya que el ruido puede deteriorar los resultados obtenidos. Las principales ventajas sobre el tradicional,se podrían enumerar y son los siguientes:

- Economía. El equipo no necesita la adquisición de excitadores y puede realizarse in situ.

- La medición se puede realizar con el equipo o estructura en funcionamiento, con lo que puede seguir estando en servicio mientras se realiza.

- Al no existir cargas externas que tengan que excitarlo evitamos el posible riesgo de 
dañar el modelo.

- Tiempo. La realización de este ensayo se ejecuta en menos tiempo que si se tuviese que excitar la estructura y validar la adquisición.

Al ser el objeto de estudio una estructura de gran tamaño y de geometría compleja se estima que el método OMA es el que mejor se adapta a esas condiciones.

A la estructura se podrán realizar mediciones in situ para validar los resultados obtenidos por el modelo de elementos finitos para esos parámetros de módulo de Young, densidad, secciones tubulares y cables. En el presente trabajo no se han podido adjuntar dichos resultados experimentales al carecer del equipo completo, pero se estima poder hacerlo en los posteriores meses a la fecha de entrega del presente trabajo.

Aplicaciones cableadas o inalámbricas necesitan una metodología planeada para emplazar los sensores. El emplazamiento de sensores puede a su vez ser permanente para hacer una monitorización fija o puede ser temporal en el caso de ser una monitorización puntual. Además, las técnicas de Monitorización de Condición Estructural (más conocido por su nombre en inglés Structural Health Monitoring y por sus siglas, SHM) requieren un número óptimo de sensores para la identificación del sistema, la identificación de daño estructural y para la actualización de modelo de elementos finitos.

\section{Motivación y Objetivos.}

El análisis modal operacional y la localización de sensores albergan actualmente un gran ámbito de aplicación en los distintos campos de la ingeniería. Se podrían citar entre otros los siguientes:

- Ingeniería aeroespacial y aeronaútica. Debido a la complejidad de dichas estructuras espaciales el análisis modal operacional resulta ideal a la hora de testear del modelo. Por dar un ejemplo, se han realizado publicaciones sobre modelización de vehículos espaciales de la agencia aeroespacial americana NASA que han pasado por técnicas de localización de sensores como las basadas en la matriz de información de Fisher, ya denominada antes FIM.

- Ingeniería estructural. En general las estructuras se estudian con OMA al ser difíciles de excitar por tamaño y por tener que hacer los ensayos in situ. Además, las técnicas de SHM también se centran en el OMA, donde una instalación de sensores permanentes puede ver el comportamiento de la estructura a través del tiempo sin ser excitadas.

- Ingeniería industrial, Diseño Industrial. Tras la dificultad de poder excitar modelos o piezas se recurre al estudio de la respuesta siguiendo estos métodos. Además, 
la incorporación al algoritmo de incertidumbres y posibilidad de actualización del modelo ha significado un avance para el diseño.

Como línea de investigación es un tema de profunda actualidad, ya que constantemente surgen nuevas publicaciones con resultados, aplicaciones y mejoras en el emplazamiento óptimo de sensores, el SHM y en parámetros de incertidumbre.

Existen numerosas publicaciones que se centran en el estudio del Análisis Modal utilizando como modelo de elementos finitos una estructura sencilla o elemento en 2D, como se puede ver en la bibliografia. La clave del presente trabajo es aplicar dichas rutinas a una estructura mucho más compleja. Esto es posible gracias a la capacidad de cálculo de los ordenadores actuales y la posibilidad de realizar iterativamente miles de operaciones de manera automática sin la necesidad de una persona física que los requiera. El principal objetivo inherente al trabajo es el de ser capaces de realizar cálculos complejos y para ello se utilizan aplicaciones y rutinas que sean capaces de trabajar automáticamente con un único llamamiento inicial.

\section{Estructura de la memoria.}

Esta memoria se estructura en varios capítulos, con la siguiente distribución de los temas trabajados:

- Capítulo 1. Se presenta el Estado del arte en el que se detalla los avances en la investigación sobre el análisis modal operacional y las publicaciones.

- Capítulo2 Se realiza una descripción concisa del modelo que hemos utilizado en este trabajo, adjuntándose fotografias y detalles del modelo de elementos finitos.

- Capítulo 3. Se definen las cuatro metodologías de localización óptima de sensores que van a ser aplicadas sobre el modelo.

- Capítulo 4. En este apartado se tratarán los resultados numéricos, representando las distintas incertidumbres en las propiedades, distintas metodologías y niveles de ruido. Se añaden gráficas con las distintas metodologías e incertidumbres para ser analizadas.

- Capítulo 5. Finalmente, se comentan las conclusiones obtenidas de los distintos métodos, así como un estudio de las líneas de trabajo futuro.

- Bibliografía. 


\section{Capítulo 1}

\section{Estado del Arte.}

En este capítulo, se introducirá de forma general el estado del arte ya que nuestro objetivo será conocer y sistematizar la producción científica en las metodologías del análisis modal operacional, optimización de las técnicas, publicaciones basadas en incertidumbre, ejercicios de localización de sensores, Structural Health Monitoring y avances con la introdución de ruido a la señal.

\subsection{Metodologías de localización de sensores.}

El emplazamiento de sensores en una estructura de acogida en un paso inicial importante en el campo del análisis modal experimental (EMA) y en particular del análisis modal operacional (OMA) [1, 2, 3]. Aplicaciones con metodologías cableadas o inalámbricas necesariamente necesitan una metodología planeada para localizar los sensores. El emplazamiento de sensores puede a su vez ser permanente para hacer una monitorización permanente o puede ser temporal en el caso de ser una monitorización de sensores itinerante. Además, las técnicas de Monitorización de Condición Estructural (SHM) requieren un número óptimo de sensores para la identificación del sistema, la identificación de daño estructural y para la actualización de modelo de elementos finitos.

Diferentes metodologías han sido desarrolladas para la colocación del sensor óptimo (OSP)[4]. Estos pueden ser clasificados principalmente en dos grupos: métodos sub-óptimos y métodos basados en estrategias de optimización formal. Los métodos sub-óptimos se basan en técnicas iterativas y estos últimos de tratar el problema como un problema de optimización clásica. Kammer[5] propone un método iterativo, el método de independencia efectiva (EFI), basado en la maximización del determinante de la matriz de información de Fisher (FIM). Esta matriz es definida como el producto de la matriz modal y su traspuesta. La idea principal de este método se basa en la independencia lineal de las formas de los modos. Se reduce el número de sensores de forma iterativa por eliminación de los grados de libertad (DOFs) de la matriz de modos que contribuyen menos 
a esta independencia. Este método produce una solución sub-óptima. Esta idea puede extenderse también a acelerómetros monoaxiales, biaxiales y triaxiales [6]. La Matriz de Fisher también puede ser medida por el uso de la matriz de masa de un modelo de elemento finitos [7]. Las técnicas de optimización de rango de matriz de energía (EMRO) son similares al método EFI pero en este caso la idea principal es aprovechar al máximo la energía de deformación de la estructura en lugar del determinante de la matriz de la FIM [8]. Este método también puede basarse en la energía cinética mediante la matriz de masa en lugar de la matriz de rigidez [9].

La identificación sistemática, como una primera etapa de SHM, requiere la colocación óptima de los sensores. Una de las primeras aplicaciones de metodologías de posicionamiento de sensores para identificación de sistemas eran las estructuras espaciales en órbita. Kammer y Yao [10] estudiaron el algoritmo EFI para colocar un número mínimo de sensores en una gran estructura espacial para la identificación de las formas modales importantes dinámicamente. Cuatro algoritmos de colocación del sensor representativo (EFI, reducción de Guyan, Driving point residue (DPR) y energía cinética) se compararon en órbita modal identificación por Glassburn y Smith [11]. Estructuras civiles y, en particular, caracterización de sistemas estructurales son otras aplicaciones de estas metodologías de las OSP. Una estrategia basada en la matriz de información de Fisher y un algoritmo rápido para las sistemas de múltiples grados de libertad (MDOFs) viene determinado en [12]. Kirkegaard y Brincker [13] presentan una publicación donde las mediciones fueron modeladas por un campo aleatorio con la función de covarianza no trivial. Además, ha sido investigada la influencia del ruido en la ubicación óptima de los sensores. Kammer [14] presentó una relación formal entre la localización de sensores según EFI y un método de realización de sistema llamado el Eigensystem realization algorithm (ERA). Papadimitriou [15] utiliza la entropía de la información, que midiendo la incertidumbre en los parámetros del sistema estructural, fue usada como medición del rendimiento de una configuración de localización de sensores. Kwon [16] estudió la aplicación de un algoritmo basado en la sensibilidad modal para determinar la localización óptima de acelerómetros para la identificación de sistemas estructurales en el dominio de la frecuencia. Sistemas estructurales de carácter civil e industrial suelen tenger un gran número de DOFs y por lo tanto sin necesarios algoritmos eficientes. Stephan [17] presenta un algoritmo sub-óptimo rápido para maximizar la observación de modo.

\subsection{Detección de daño estructural.}

Las técnicas de detección de daño estructural basadas en la vibración dependen principalmente del número óptimo de sensores y su localización para obtener abundante información de una estructura [18]. SKjaerbaek y Nielsen [19] presentan un método para la localización de daños estructurales en una estructura de hormigón excitada sísmicamente, con especial énfasis en la óptima ubicación de los sensores. Worden y Burrows [20] describen un método para detección de fallas y clasificación utilizando redes neuronales de optimización combinatoria. Kripakaran y Smith [21] implementan una aplicación de 
ubicaciones de los sensores para la detección de daños para el caso de un puente de ferrocarril. Hong et al. [22], presentan una técnica de colocación del sensores para detectar la longitud de una grieta de las estructuras de vehículos. Vanli [23] et al estudiaron un problema de optimización de mínimo-máximo con el objetivo de encontrar las coordenadas de un número determinado de sensores. El objetivo considerado en este estudio fue minimizar el máximo probabilidad de la no detección de la red de sensores. También se emplean métodos de optimización en colocación de sensores para la detección de daños. Raich y Liszkai [24] desarrollaron una optimización multi-objetivo de capas de excitación y sensor para la identificación del daño en base a la función de respuesta en frecuencia. Se mostraron estudios similares sobre la OSP para detección de daño estructural en [25, 26].

\subsection{Actualización del modelo.}

Los procedimientos para actualizar el modelo de elementos finitos [27], una parte del proceso de SHM, también pueden mejorarse por metodologías de localización óptima de sensores. Reynier y Kandil [28] propusieron una reducción del modelo para el procedimiento de actualización complementada con dos enfoques: el primero se basaba en la minimización de los efectos del ruido y la segunda surgieron desde una perspectiva de control basado en la observación de la matriz de Gram. Papadimitriou y Beck [29] emplean una localización óptima de sensores basada en la entropía para la actualización del modelo estructural. Lam et al [30], revisa el problema de la instalación de sensores para la actualización de modelo estructural dentro de un marco estadístico bayesiano.

\subsection{Parámetros de incertidumbre.}

En los últimos años, la investigación se centra en la incorporación de las incertidumbres asociadas con estructuras reales en la simulación numérica para predicciones fiables. Las incertidumbres pueden clasificarse principalmente como incertidumbre paramétrica y de la forma del modelo [31, 32]. La incertidumbre paramétrica se asocia con las discrepancias entre los valores reales de los sistemas físicos y los parámetros de entrada utilizados para el análisis. Las incertidumbres de la forma del modelo están asociadas con la falta de modelado preciso del sistema físico. Sin embargo, en este trabajo, se consideran sólo las incertidumbres paramétricas. Los análisis de incertidumbre hacen uso de muestreo estadístico, comprobación de hipótesis y análisis de datos de entrada-salida para caracterizar el efecto de las incertidumbres de forma paramétrica y modelo en los experimentos estructurales o en las simulaciones numéricas [33, 34, 35, 36]. La incertidumbre puede alterar la localización óptima de sensores y a posteriori afectan al SHM. Muy pocos estudios han considerado la ubicación óptima de sensores con incertidumbre paramétrica. Guratzsch y Mahadevan desarrollaron una metodología para el diseño de una matriz óptima de sensores de SHM bajo incertidumbre. En este papel, métodos de cuantificación de incertidumbre bajo cargas mecánicas y térmicas transitorias se emplean 
para validar un modelo de elementos finitos con datos experimentales. Azarbayejani et al. [37], presentan un enfoque probabilístico para determinar el número de sensores y su localización óptimas para SHM. 


\section{Capítulo 2}

\section{Modelo de Elementos Finitos.}

En este capítulo se presenta el modelo que se toma como objeto de estudio. El diseño elegido es una cubierta para un recinto multifuncional abierto con cuatro paraboloides hiperbólicos textiles, que cubre una superficie de 46x24 metros. La peculiaridad de la misma es que los cuatro soportes del conjunto no están situados en cada esquina, sino que reside en los puntos medios de los lados. Esto implica considerar cuatro alas en voladizo con 23 y 12 metros. La elección de esta estructura se debe a la complejidad de la misma, ya que no es una estructura convencional.Los autores describieron la obra en una publicación [38] y existe un estudio acústico sobre la estructura [39].

\subsection{Precedentes.}

Antes de hablar de la estructura se van a tratar los precedentes de la misma. Las estructuras de paraboloides hiperbólicos surgieron a través del arquitecto Félix Candela (1910-1997), que desde el inicio de su carrera se centró en la realización de estructuras con cubiertas de esta geometría. La singularidad de las mismas y los escasos medios para el cálculo por falta de herramientas informáticas hacen de él y su obra un hito en la historia de la arquitectura. Son composiciones que funcionan muy bien cuando se requiere cubrir espacios amplios, tienen una gran facilidad constructiva al tener los bordes rectos y el resultado hace que sean llamativas al no ser convencionales. En el caso de ser de hormigón los espesores que pueden conseguirse son realmente delgados, siempre dependiendo de la luz a cubrir.

Algunos ejemplos de obras de paraboloides hiperbólicos de Félix Candela son el $\mathrm{Pa}$ bellón de Rayos Cósmicos (1951) , Almacén de Herdez (1955-1956) ,Capilla de Nuestra Señora de la Soledad (1956), el Restaurante de Xochimilco (1957-1958), la Estación de metro de San Lázaro (1969) y su obra póstuma Oceanografic, Ciudad de las Artes y las Ciencias en Valencia (2002). No sólo Candela ha trabajado con esta superficie para construir, pero si bien es cierto que fue el precursor que hizo que pareciese fácil hacerlo. 


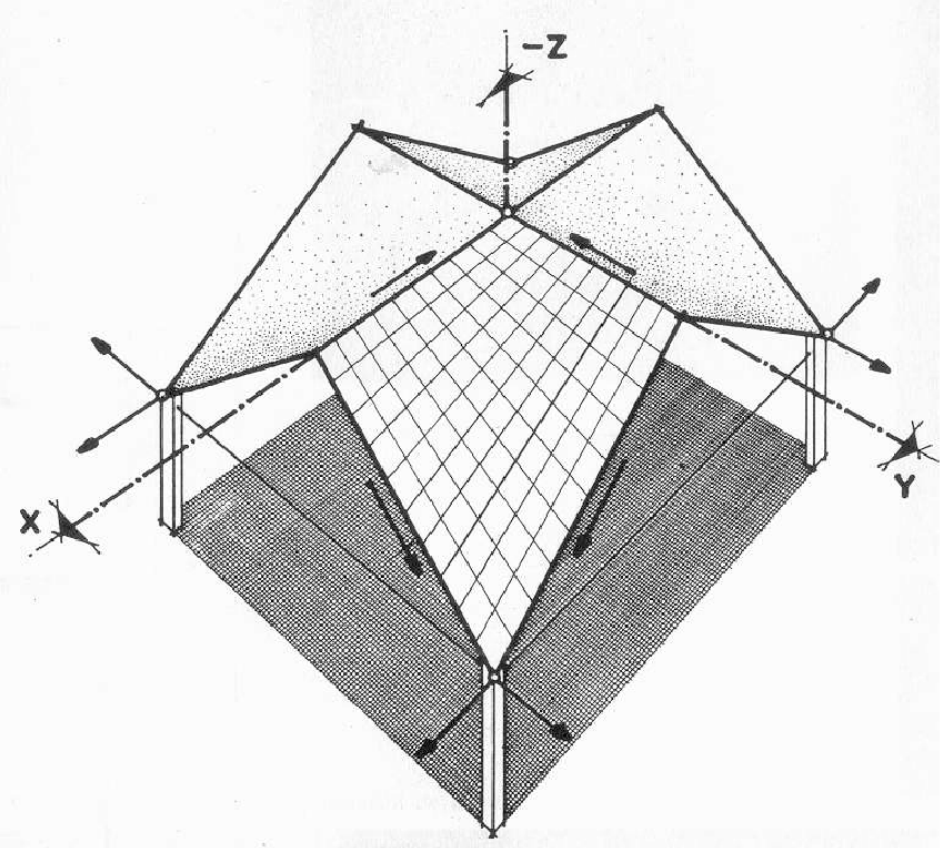

Figura 2.1: Almacén de Herdez, Mexico D.F., 1955-1956. Félix Candela.

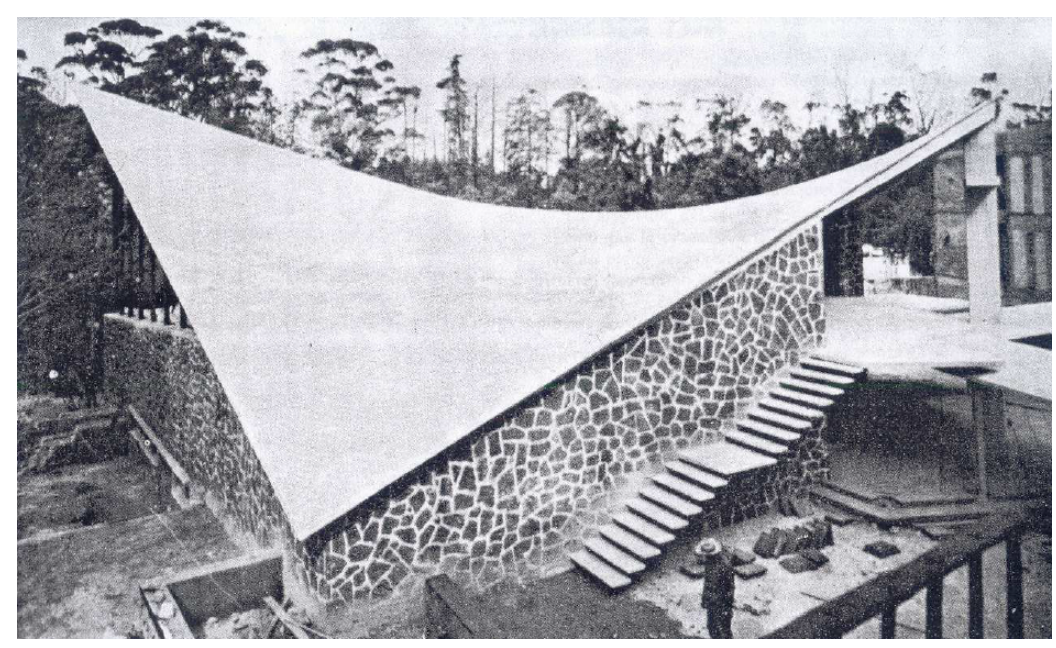

Figura 2.2: Capilla de Nuestra Señora de la Soledad, Mexico D.F., 1956. Félix Candela. 


\subsection{Descripción de la estructura.}

El proyecto está emplazado en el Centro de Alto Rendimiento de la Cartuja, en Sevilla, donde fue construido en enero de 2010. La estructura tenía una serie de requisitos que cumplir, unos puntos de partida en los que los arquitectos debían apoyarse para obtener la mejor solución al problema. Precisamente dichas condiciones que la estructura debía cumplir hacían que fuese un proyecto muy exigente. Podemos enumerar:

- Temporalidad. El proyecto deberia ser una estructura de carácter eventual, segun la normativa urbanística. No se permitía la construcción de ningún elemento permanente.

- Grandes luces. Al ser una pista polideportiva, la superficie que se debia cubrir era de 40×20 más un margen de juego que finalmente quedaría en una cubierta de 46×24 metros, un total de $\mathbf{1 1 0 4} \mathbf{~ m}^{2}$ de superficie.

- Cerramiento retráctil. La pista no estaba planteada para tener una grada, sino que como condición en el encargo se estimaba un cerramiento de tipo cortina que nos obligaria a tener una viga horizontal de rodadura.

Una vez tenemos los datos de partida, se propone realizar una estructura de acero ya que es la mejor forma de instalar una estructura que puede ser retirada y pudiese ejecutarse en otro lugar sin ser derruida. Los paños que tendrán la forma de paraboloide hiperbólico se compondrán de un tejido de material compuesto de PVC.

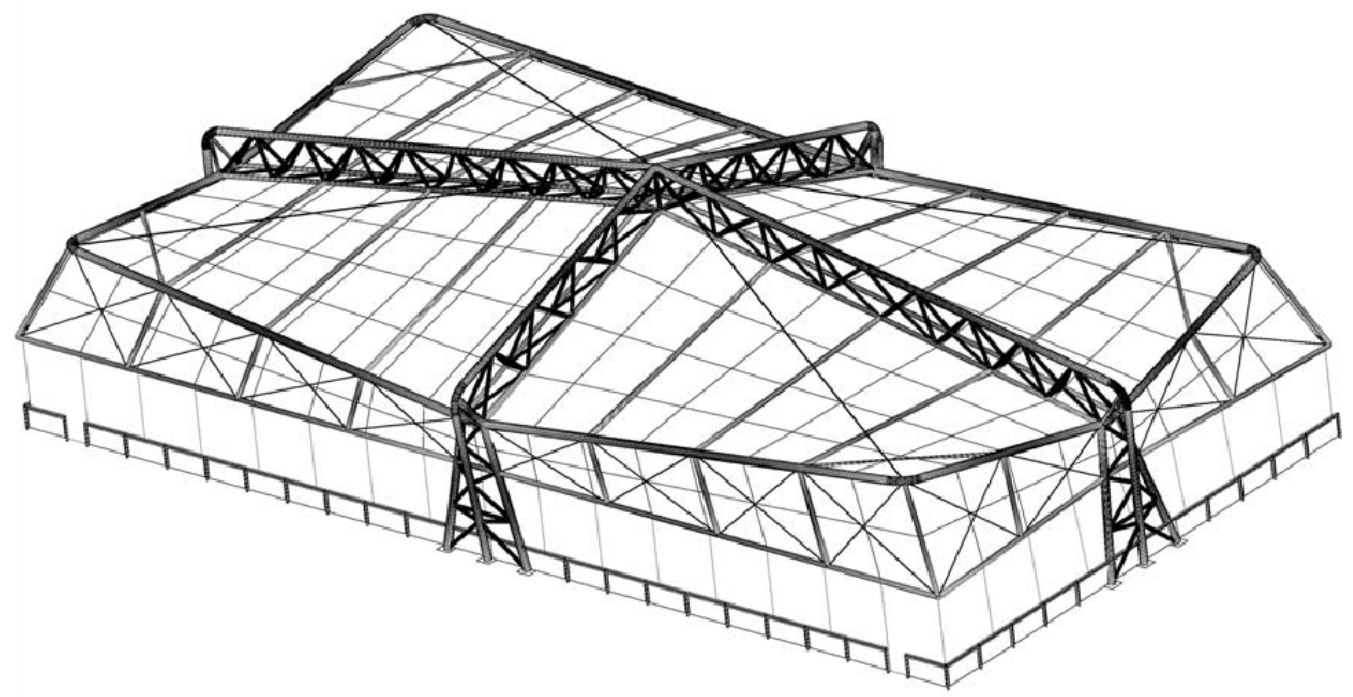

Figura 2.3: Propuesta para cubrir recinto polideportivo de 46x24 m.

Para resolver constructivamente el proyecto se aportaron las disposiciones constructivas capaces de dar solución a todos los aspectos que consolidaran su funcionamiento. 
Capítulo 2. Modelo de Elementos Finitos.

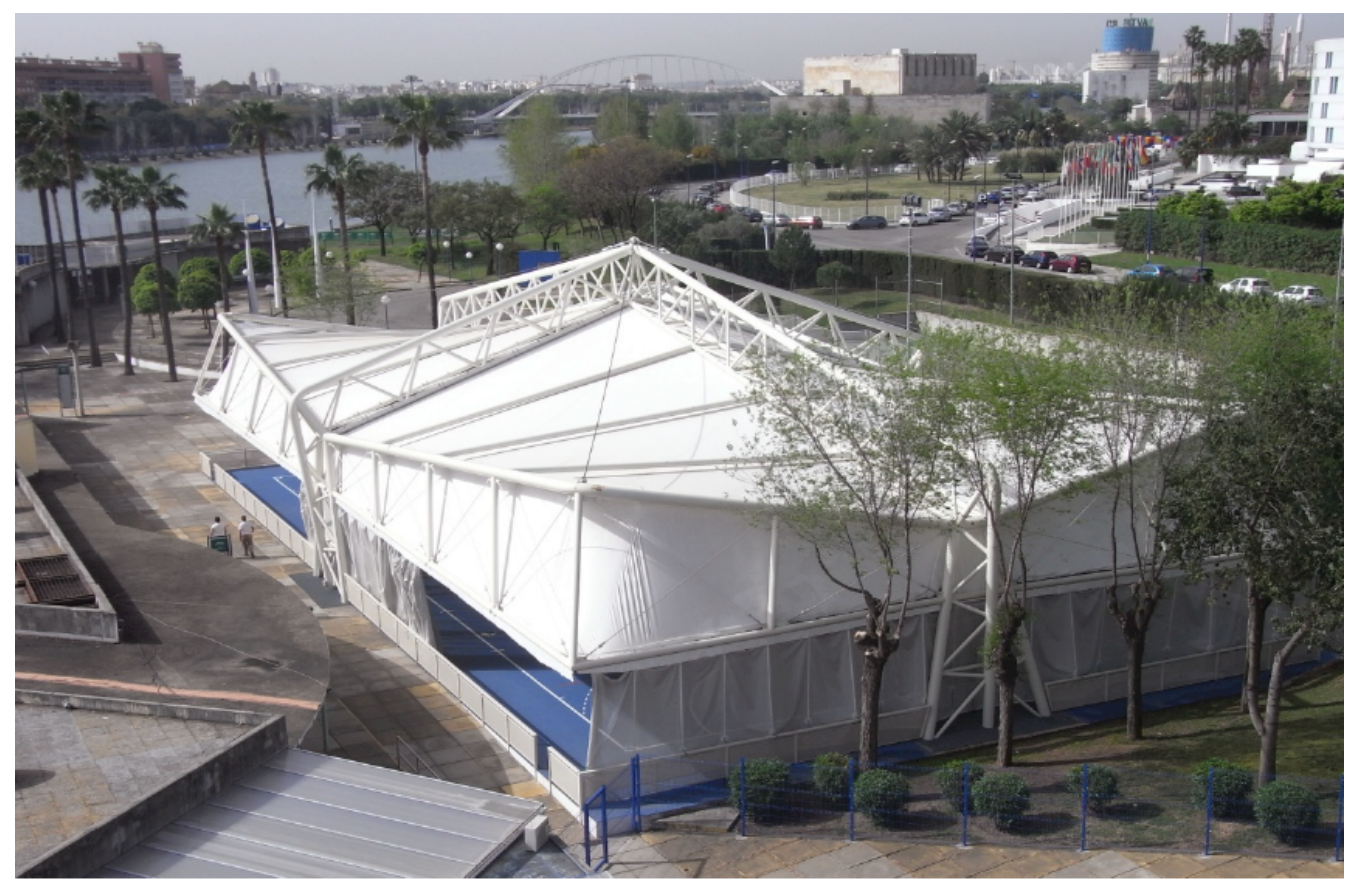

Figura 2.4: Vista general.

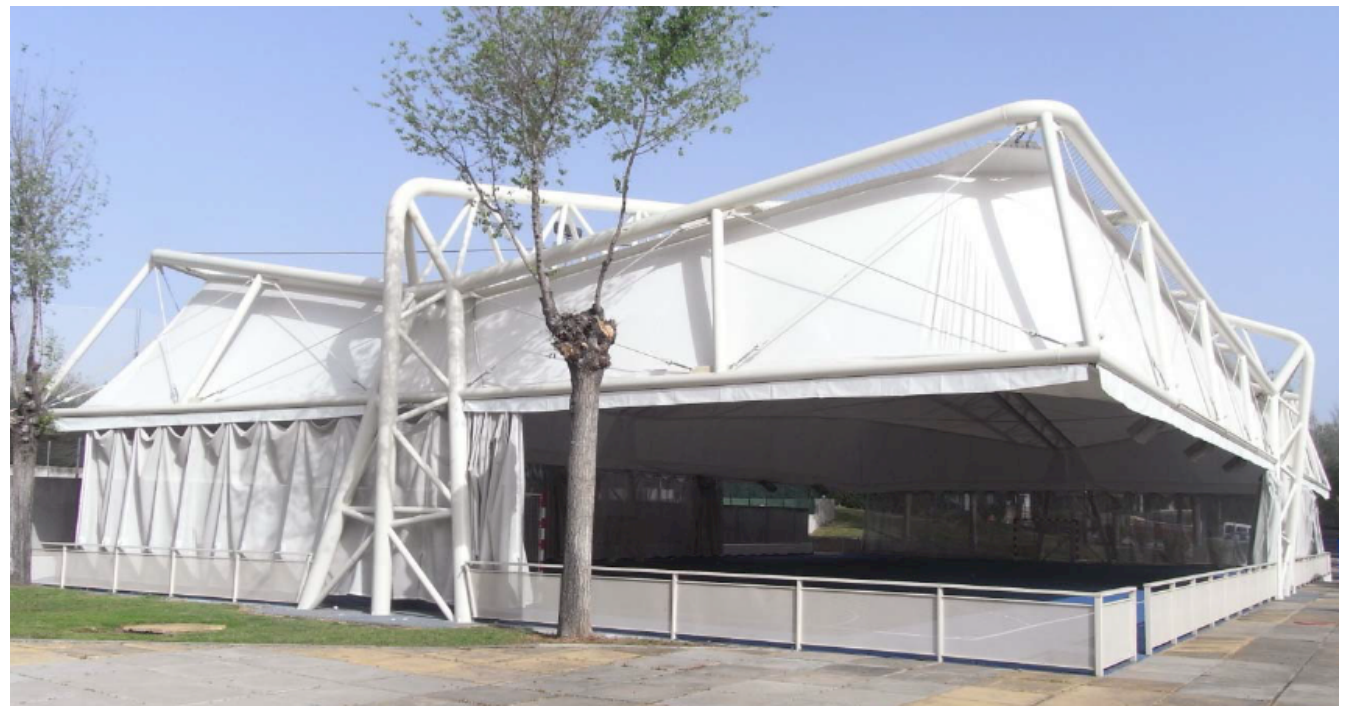

Figura 2.5: Vista lateral con cerramiento retráctil.

Como podemos ver en la Figura 2.3 la peculiaridad de la estructura reside en los apoyos que se encuentran en los puntos medios de los lados. Esto obliga a reforzar el marco estructural principal con cables estabilizadores en los recuadros de la estructura lateral. 


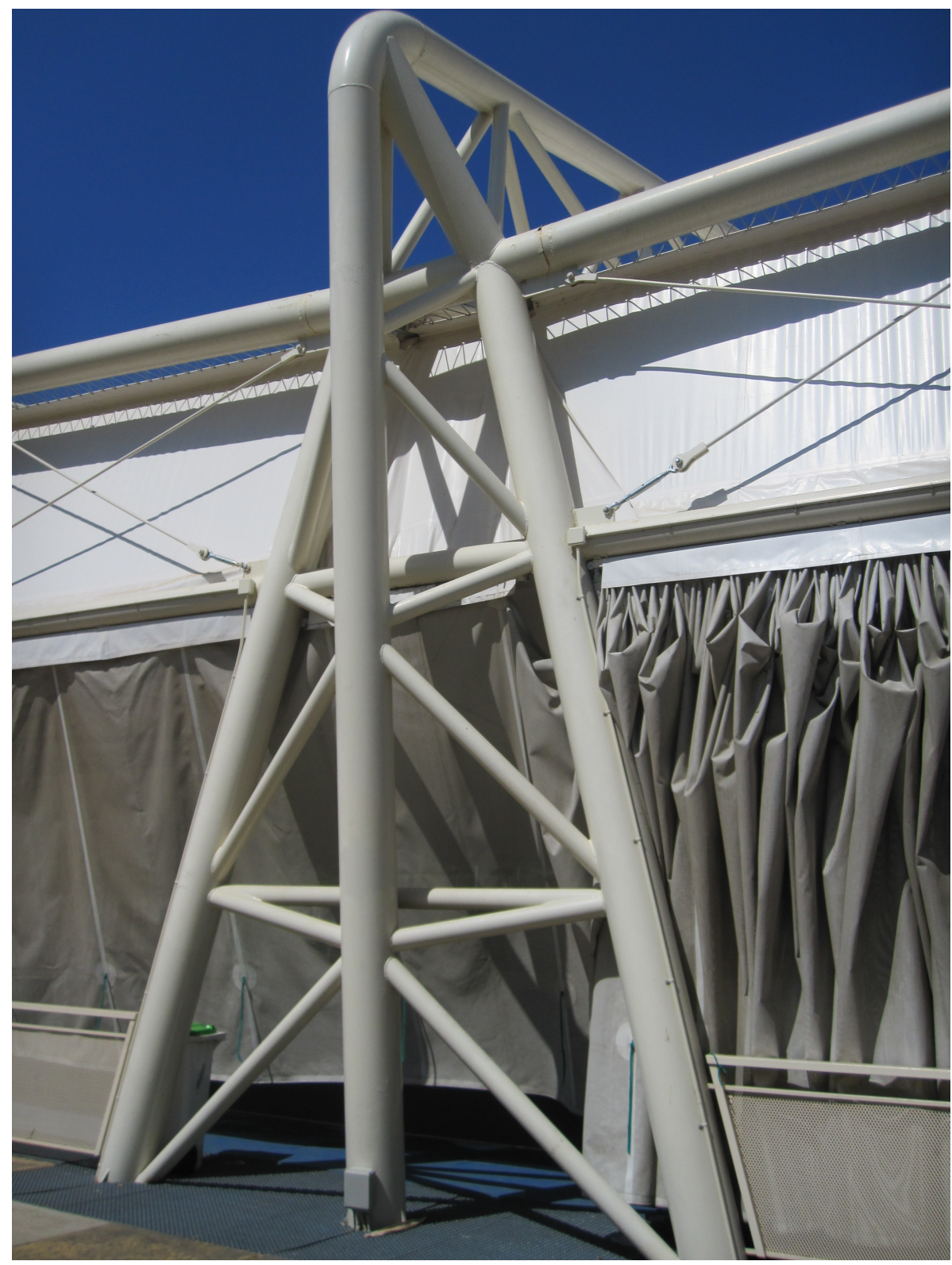

Figura 2.6: Detalle de soporte lateral.

Además para poder salvar las luces se recurre a una vigas centrales. Se introduce un sistema de ventilación en cumbrera para evitar condensaciones en el interior de la superficie de cubierta y proporcionar un flujo de aire permanente y un cerramiento de trama abierta deslizante sobre guías, que puede ser retirado o extendido fácilmente.

Con respecto al proceso de ejecución de la estructura, se realizó casi en su totalidad en taller, siendo un proceso que requirió medios especiales. Los soportes se ejecutaron 


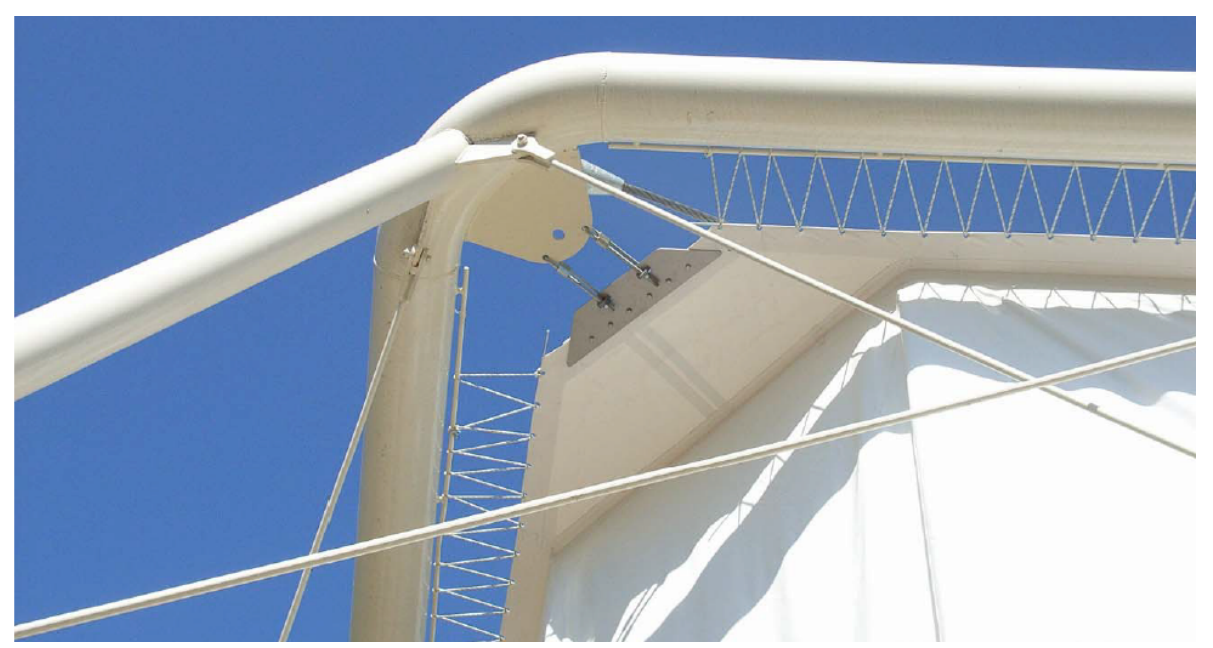

Figura 2.7: Detalle de encuentro entre la lona y el marco estructural.

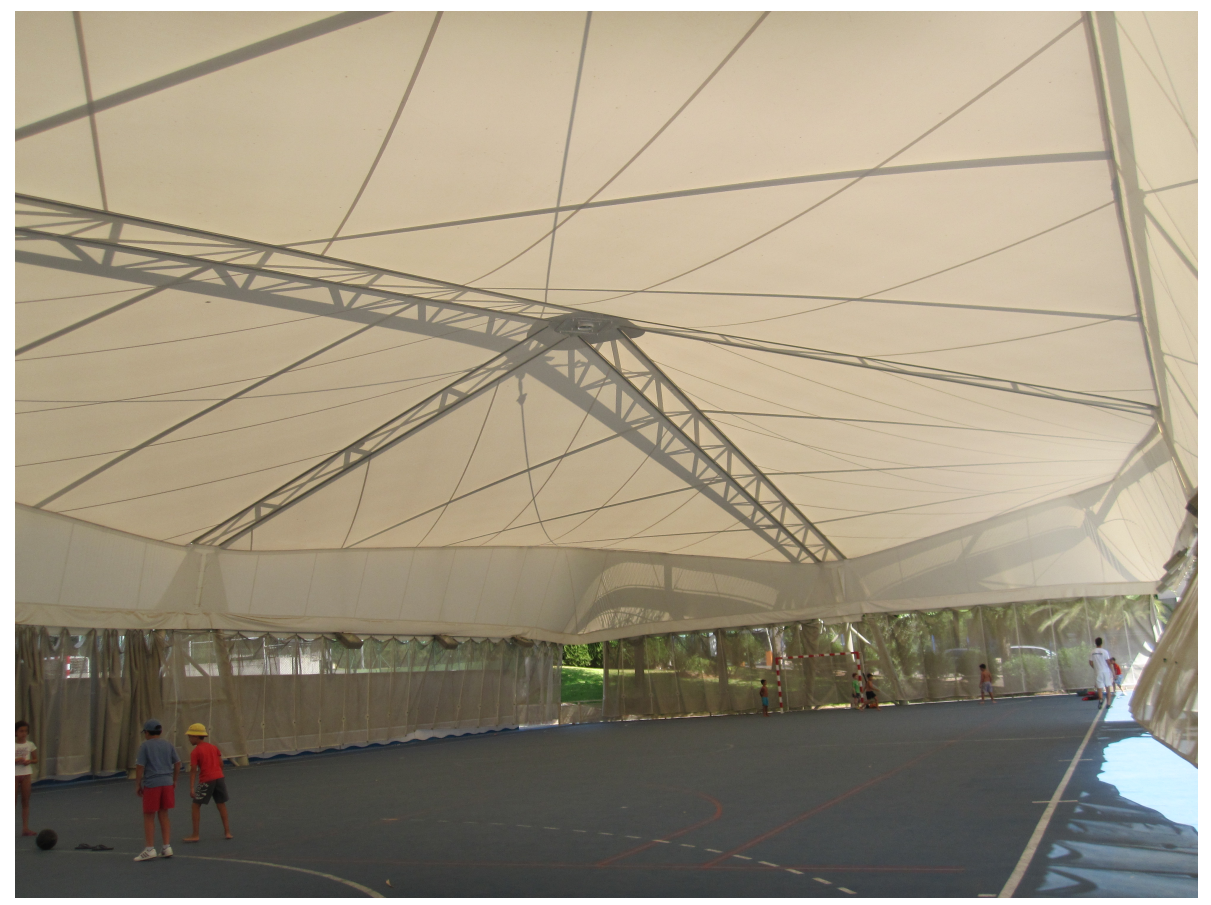

Figura 2.8: Vista del interior.

completos en taller y se realizó el pintado de los mismos. El transporte a la obra tuvo que realizarse con camiones especialmente largos.

Una vez colocada la estructura se procedió a instalar la lona textil. Por el debido peso de la misma, de $\mathbf{1 3 0 0} \mathrm{g} / \mathrm{m}^{2}$, se ejecutó en cuatro partes para facilitar la construcción. 


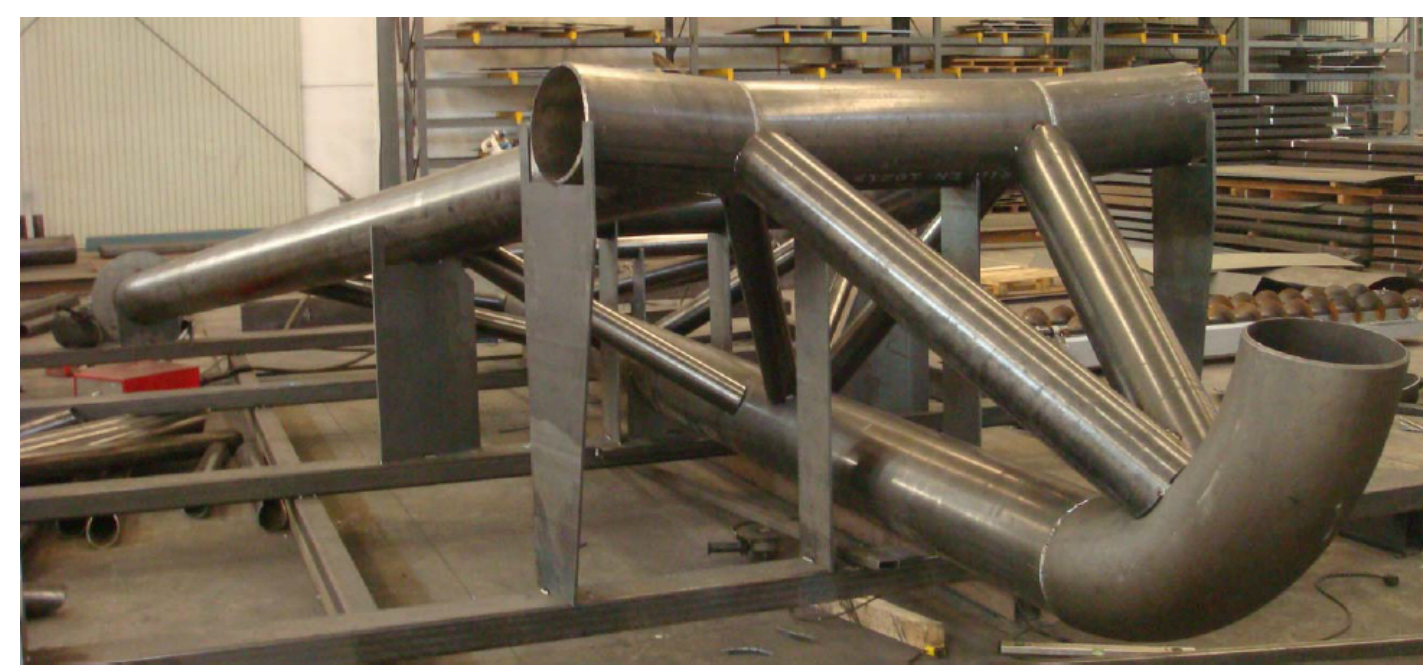

Figura 2.9: Proceso de fabricación en taller.

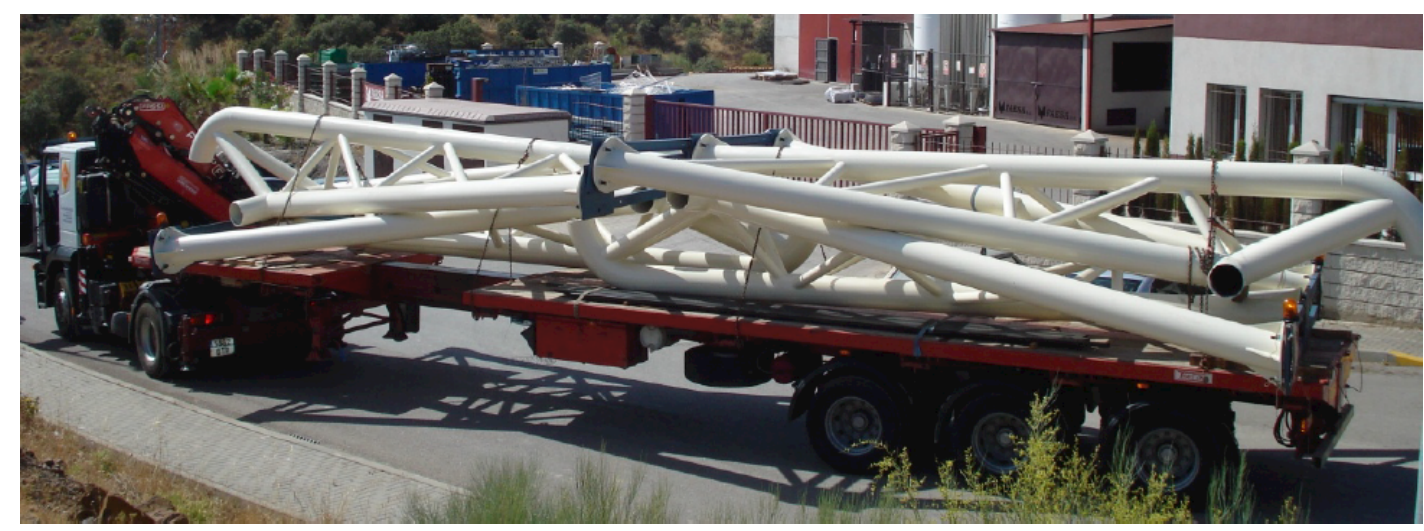

Figura 2.10: Transporte de soportes.

\subsection{Cálculo en SAP2000.}

La estructura se modelizó y calculó en SAP2000. Fue necesario introducir tres codales que partieran la superficie para evitar la flexión lateral de las cerchas de borde y unos codales en las esquinas a $45^{\circ}$. En el cuadro 2.1 se muestran los perfiles utilizados, mostrando en la figura 2.11 en distinto color los distintos elementos.

La estructura tiene en su totalidad 190 nodos, de los que se obtienen 570 grados de libertad traslacionales. Los datos que se necesitan de la estructura son arrojados a la hora de solicitar el análisis modal de la misma. El programa SAP2000 detallará una serie de archivos de texto que determina la numeración interna de los grados de libertad por nodos ( $\mathrm{ux}, \mathrm{uy}, \mathrm{uz}, \theta \mathrm{x}, \theta \mathrm{y}, \theta \mathrm{z}$ ), las matrices de masas y de rigidez. Además detalla la matriz 


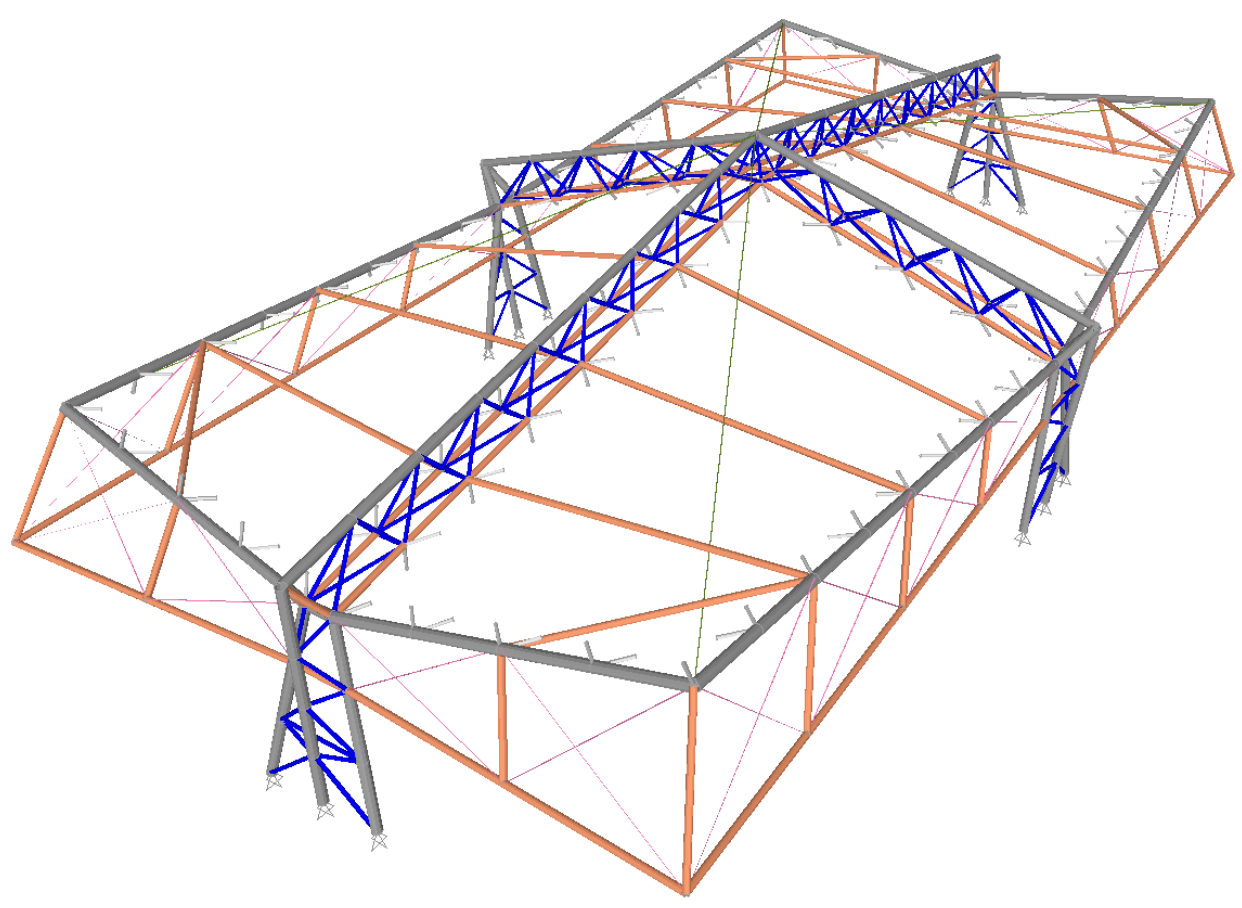

Figura 2.11: Estructura modelizada.

\begin{tabular}{lccc}
\hline Nombre & Diámetro $\varnothing$ & Espesor & Color \\
\hline Tubular1 & $323 \mathrm{~mm}$ & $8 \mathrm{~mm}$ & Gris \\
Tubular2 & $200 \mathrm{~mm}$ & $6 \mathrm{~mm}$ & Naranja \\
Tubular3 & $120 \mathrm{~mm}$ & $5 \mathrm{~mm}$ & Azul \\
Cable1 & $36 \mathrm{~mm}$ & $17,5 \mathrm{~mm}$ & Verde \\
Cable2 & $25 \mathrm{~mm}$ & $12 \mathrm{~mm}$ & Morado \\
\hline
\end{tabular}

Cuadro 2.1: Secciones utilizadas en proyecto.

de formas modales y las frecuencias naturales de los primeros modos de la estructura.

\subsection{Introducción de datos en Matlab.}

Para trabajar con los datos de la estructura de SAP2000,se tendría que introducir los mismos en un programa de lenguaje matemático para posibilitar la ejecución de rutinas de análisis modal operacional de manera rápida. Además, en el caso de tener que calcular miles de iteraciones, que es el hándicap del trabajo, hacerlo lo más rapido posible va a 
facilitar la viabilidad para el cálculo de estructuras más complejas. Para ello se procede a usar la aplicación API de SAP2000, que posibilita traducir los datos a lenguaje Matlab para poder trabajarlo. Esta aplicación de SAP2000 es reciente y poco conocida pero a la vez es muy útil y significa un gran avance para posibilitar el trabajo en rutinas. 
Capítulo 2. Modelo de Elementos Finitos. 


\section{Capítulo 3}

\section{Metodologías de localización de sensores.}

En esta sección se van a considerar cuatro metodologias de optimización de sensores que son comunmente usadas en la localización óptima de sensores (OSP).Las dos basadas en la Matriz de información de Fisher (EFI Y EFIWM) y las otras dos basadas en el concepto de optimización del rango de la Matriz de energía (KEMRO Y SEMRO).

\subsection{Método de Independencia Efectiva (EFI).}

Kammer [5] introdujo el algoritmo de localización óptima de sensores EFI que pretende buscar el mejor conjunto de localización de DOFs desde todas las opciones candidatas en la estructura de tal forma que se mantenga la independencia lineal de las formas modales. El punto de partida de este método es la matriz de modos completa de un modelo de elementos finitos. Todos los DOFs del modelo de elementos finitos no se pueden medir en la estructura real debido a limitaciones físicas. Por lo tanto, los DOFs correspondientes a las rotaciones y coordenadas que no pueden ser medidas son eliminados de la matriz modal completa. Las filas correspondientes a DOFs que pueden ser medidos se mantienen y las columnas correspondientes a modos a buscar se conservan en la matriz completa modal. La matriz de información de Fisher (FIM) se define como:

$$
\mathbf{F I M}=\phi^{T} \phi
$$

Teniendo las siguientes propiedades:

- Es una matriz simétrica. 
- Es una matriz semidefinida, esto es, su determinante es siempre positivo y todos los autovalores son también positivos.

- Si el vector columna (las formas modales) son linealmente independientes, el rango total es igual a la dimensión de la matriz, esto es el número de formas modales buscadas.

Si el determinante de la FIM es cero, las columnas de la matriz modal (es decir, los modos elegidos) son linealmente dependientes. Por lo tanto, es el propósito del método EFI seleccionar los mejores DOFs (para colocar los sensores) que maximizan el determinante de la FIM. Los DOFs para ser eliminados de la matriz modal son elegidos de forma iterativa. La matriz de proyección ortogonal se calcula como:

$$
\mathbf{E}=\phi \mathbf{F I M} \mathbf{M}^{-1} \phi^{T}
$$

Cada elemento de la diagonal de la matriz E representa la contribución fraccional del i-ésimo DOF al rango de esta matriz y en particular la independencia lineal de la formas modales. La matriz E es una matriz cuyo rango es igual a la suma de los términos de la diagonales. El proceso de selección se puede resumir en los siguientes pasos:

1. Los DOFs que no se puede medir físicamente son eliminados. Las filas correspondientes a DOFs desde la matriz modal se eliminan.

2. Los Modos buscados se seleccionan de los de interés. Sólo las columnas de la matriz modal que corresponden a los modos que son nuestro objetivo se conservan.

3. La matriz E se calcula. La diagonal de la matriz E está ordenada de los elementos superiores a las inferiores. Se elimina el DOF con el menor valor de la matriz modal.

4. Este proceso se repite hasta que el número de DOFs que quedan es igual al número de sensores deseado.

5. En cada paso el determinante de la matriz FIM determina la evolución del proceso. Normalmente se representa como el porcentaje del valor inicial.

Este procedimiento para el método de localización óptima de sensores EFI produce una óptima solución de forma iterativa. Una versión del método EFI (EFIWM) también es estudiada en el recurso bibliográfico [40].

En este caso las matrices FIM y E corresponden a:

$$
\mathbf{F I M}=\phi^{T} \mathbf{M} \phi
$$


y por lo tanto:

$$
\mathbf{E}=\sqrt{\mathbf{M}} \phi \mathbf{F I M} \mathbf{M}^{-1} \phi^{T} \sqrt{\mathbf{M}}
$$

Donde $\mathrm{M}$ representa la matriz simétrica de masa del modelo de elementos finitos. En este caso, la reducción de Guyan se implementa en cada iteración para reducir la matriz de masa a los DOFs. Más detalles de la ponderación de masa se explican en el recurso bibliográfico [41].

\subsection{Optimización del rango de la matriz de energía (EMRO).}

La idea básica que subyace del algoritmo EMRO es lograr una configuración de localización de sensores conforme que maximiza la energía de deformación (SE) de las formas modales medidas en la estructura [8]. La energía de deformación es:

$$
\mathbf{S E}=\phi^{T} \mathbf{K} \phi
$$

donde $\mathrm{K}$ es la matriz de rigidez del modelo de elementos finitos. Este método es una versión del método EFI donde la matriz FIM es ensamblada usando la descomposición de Cholesky de la matriz de rigidez, siendo:

$$
\mathbf{K}=\mathbf{C}^{T} \mathbf{C}
$$

Donde $C$ es una matriz triangular superior. En este caso, la matriz es definida como:

$$
\mathbf{F I M}_{S E}=\psi^{T} \psi \quad \text { donde } \quad \psi=\mathbf{C} \phi
$$

La matriz E es calculada de la misma forma que el método EFI pero con una modificación, es:

$$
\mathbf{E}_{S E}=\psi \mathbf{F I M} \mathbf{M}_{S E}^{-1} \psi^{T}
$$

Una vez la matriz E es definida, el procedimientos es el mismo que para el algoritmo EFI, pero empleando la reducción de Guyan para reducir la matriz de rigidez en cada paso. Este método es usualmente llamado SEMRO. Similar a esta metodología basada en la energía de deformación, la energía cinética (KE) puede ser usada [9], dando:

$$
\mathbf{K E}=\phi^{T} \mathbf{M} \phi
$$


Este método es llamado KEMRO. Siguiendo el procedimiento de la ecuación 3.7, la matriz de masa es descompuesta por la factorización de Cholesky:

$$
\mathbf{M}=\mathbf{D}^{T} \mathbf{D}
$$

Y la matriz FIM es por tanto:

$$
\mathbf{F I M}_{K E}=\psi^{T} \psi \quad \text { donde } \quad \psi=\mathbf{D} \phi
$$

El siguiente paso es el mismo que en el caso del algoritmo SEMRO pero con la matriz $\mathbf{E}_{K E}$, dada de la siguiente forma:

$$
\mathbf{E}_{K E}=\psi \mathbf{F} \mathbf{I} \mathbf{M}_{K E}^{-1} \psi^{T}
$$

Basada en el estudio del método KEMRO dado en las referencias [42, 43]. 


\section{Capítulo 4}

\section{Resultados.}

En este punto se estudian los resultados obtenidos a través del cálculo en Matlab de las metodologías descritas, tanto de manera determinista como probabilista. Se tratan las incertidumbres aplicadas al modelo y las soluciones obtenidas tras el cálculo de los algoritmos. Como se ha mencionado previamente, se introducirán parámetros como la sección, el módulo de Young y la densidad como incertidumbres a traves de una simulación de Monte Carlo.

\subsection{Cálculo determinista de las OSP.}

\subsubsection{Búsqueda de DOFs}

Se tiene como punto de partida los valores de la estructura existentes, que recordamos son los siguientes en el cuadro 4.4 .

\begin{tabular}{lcc}
\hline Perfiles & Diámetro $\varnothing$ & Espesor \\
Tubular1 & $323 \mathrm{~mm}$ & $8 \mathrm{~mm}$ \\
Tubular2 & $200 \mathrm{~mm}$ & $6 \mathrm{~mm}$ \\
Tubular3 & $120 \mathrm{~mm}$ & $5 \mathrm{~mm}$ \\
\hline Módulo de Elasticidad & $200 \mathrm{GPa}$ & \\
\hline Densidad del acero & $7850 \mathrm{Kg} / \mathrm{m}^{3}$ & \\
\hline
\end{tabular}

Cuadro 4.1: Datos del modelo a introducir en las metodologías de OSP

Para esos datos se procede a ejecutar los cuatro algoritmos, de las que se obtienen una localización óptima de 11 sensores. En la tabla siguiente se resumen los 11 grados de libertad solución de los 570 existentes y a que nodo y eje se refiere.

Cada configuración selecciona distintos grados de libertad, y son pocas las coinciden- 
Capítulo 4. Resultados.

\begin{tabular}{|cc|cc|cc|cc|}
\multicolumn{2}{c|}{ EFI } & \multicolumn{2}{|c|}{ EFIWM } & \multicolumn{2}{c|}{ SEMRO } & \multicolumn{2}{c|}{ KEMRO } \\
DOFs & Nodo y eje & DOFs & Nodo y eje & DOFs & Nodo y eje & DOFs & Nodo y eje \\
87 & $29 \mathrm{z}$ & 408 & $136 \mathrm{z}$ & 408 & $136 \mathrm{z}$ & 408 & $136 \mathrm{z}$ \\
114 & $38 \mathrm{z}$ & 411 & $137 \mathrm{z}$ & 411 & $137 \mathrm{z}$ & 411 & $137 \mathrm{z}$ \\
141 & $47 \mathrm{z}$ & 419 & $140 \mathrm{y}$ & 415 & $139 \mathrm{x}$ & 438 & $146 \mathrm{z}$ \\
146 & $49 \mathrm{y}$ & 422 & $141 \mathrm{y}$ & 421 & $141 \mathrm{x}$ & 441 & $147 \mathrm{z}$ \\
161 & $54 \mathrm{y}$ & 438 & $146 \mathrm{z}$ & 425 & $142 \mathrm{y}$ & 485 & $162 \mathrm{y}$ \\
168 & $56 \mathrm{z}$ & 441 & $147 \mathrm{z}$ & 428 & $143 \mathrm{y}$ & 488 & $163 \mathrm{y}$ \\
456 & $152 \mathrm{z}$ & 486 & $162 \mathrm{z}$ & 430 & $144 \mathrm{x}$ & 516 & $172 \mathrm{z}$ \\
462 & $154 \mathrm{z}$ & 489 & $163 \mathrm{z}$ & 438 & $146 \mathrm{z}$ & 566 & $189 \mathrm{y}$ \\
465 & $155 \mathrm{z}$ & 535 & $179 \mathrm{x}$ & 441 & $147 \mathrm{z}$ & 568 & $190 \mathrm{x}$ \\
545 & $182 \mathrm{y}$ & 536 & $179 \mathrm{y}$ & 536 & $179 \mathrm{y}$ & 569 & $190 \mathrm{y}$ \\
548 & $183 \mathrm{y}$ & 539 & $180 \mathrm{y}$ & 543 & $181 \mathrm{z}$ & 570 & $190 \mathrm{z}$
\end{tabular}

Cuadro 4.2: DOFs y nodos solución de las distintas metodologías

cias. Si es cierto que al ser una estructura simétrica, algunos puntos escogidos por un método pueden encontrarse en otro punto simétrico a éste. Se puede ver en una visualización en 3D los DOFs que obtenemos para cada metodologia en las figuras 4.6, 4.2, 4.3 y 4.4

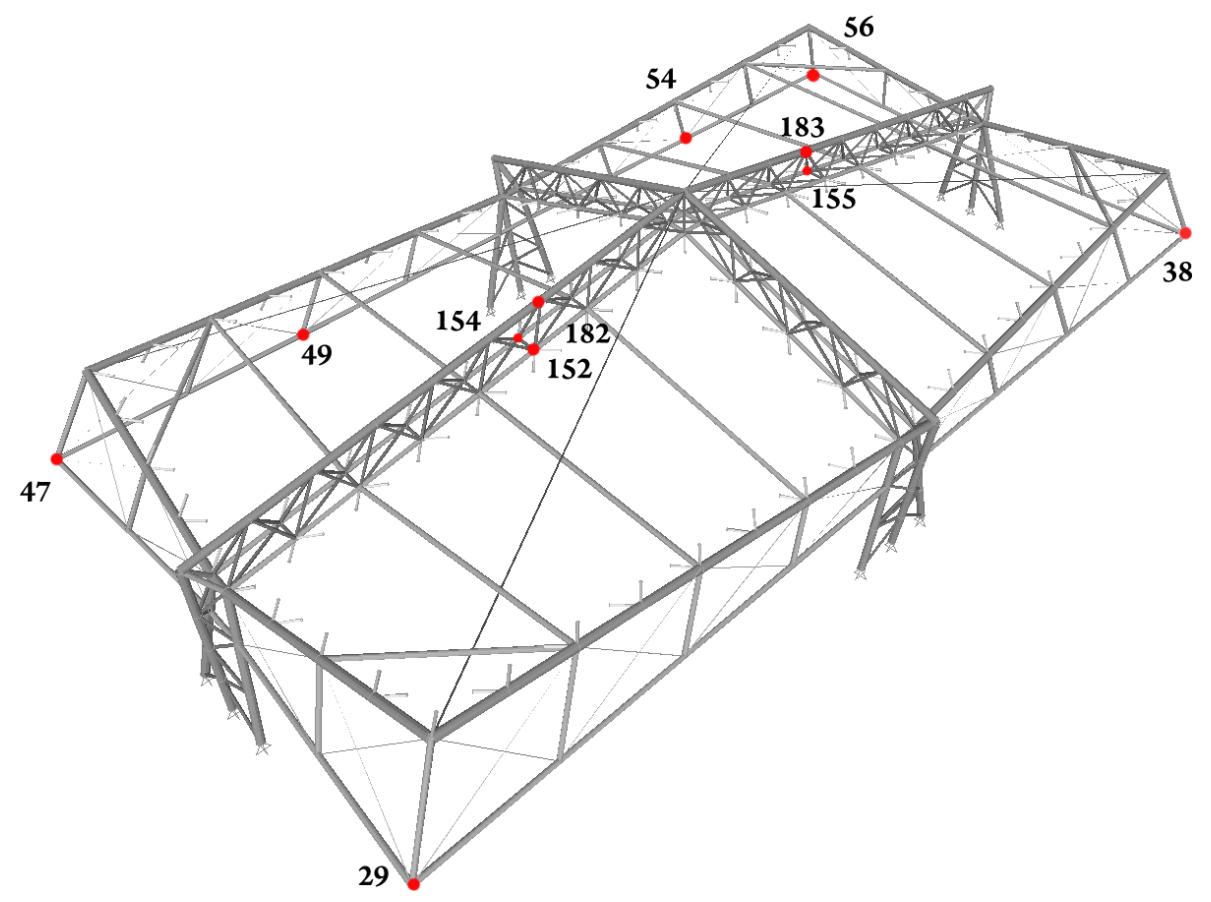

Figura 4.1: OSP obtenida para el método EFI 


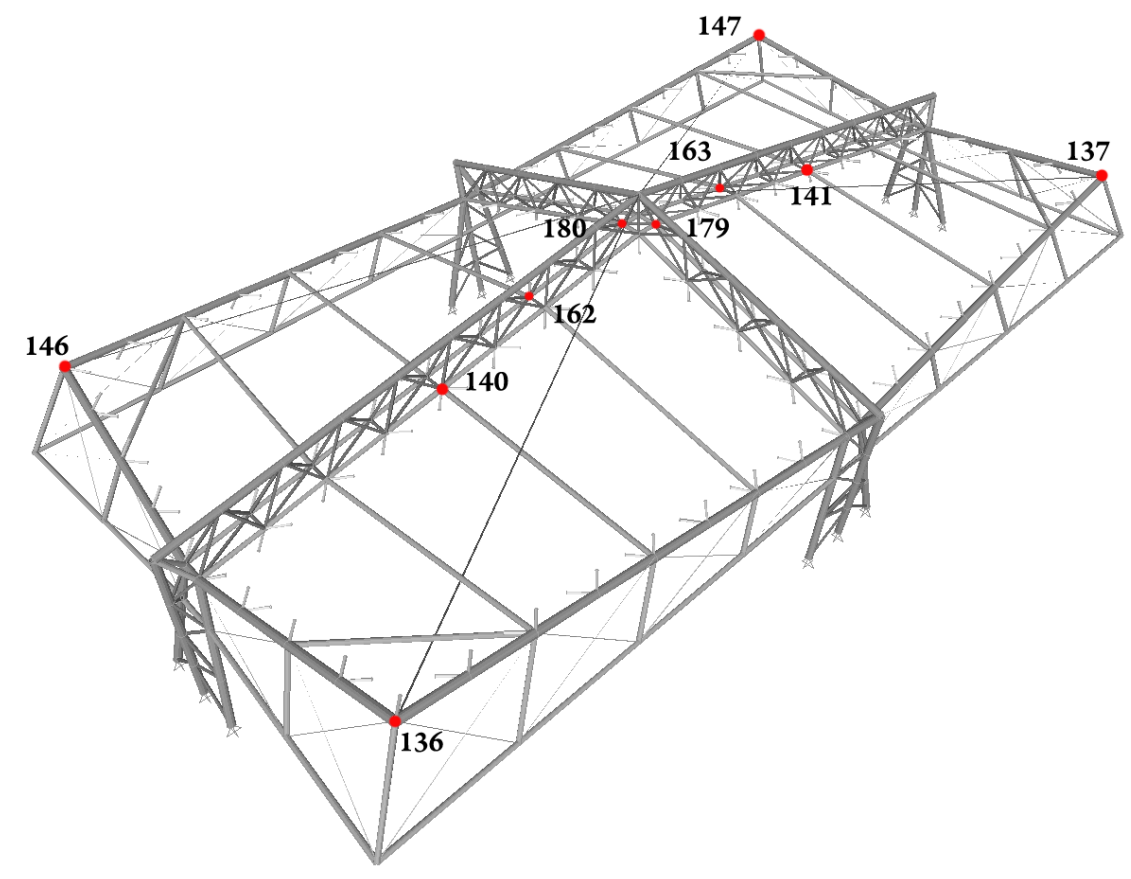

Figura 4.2: OSP obtenida para el método EFIWM

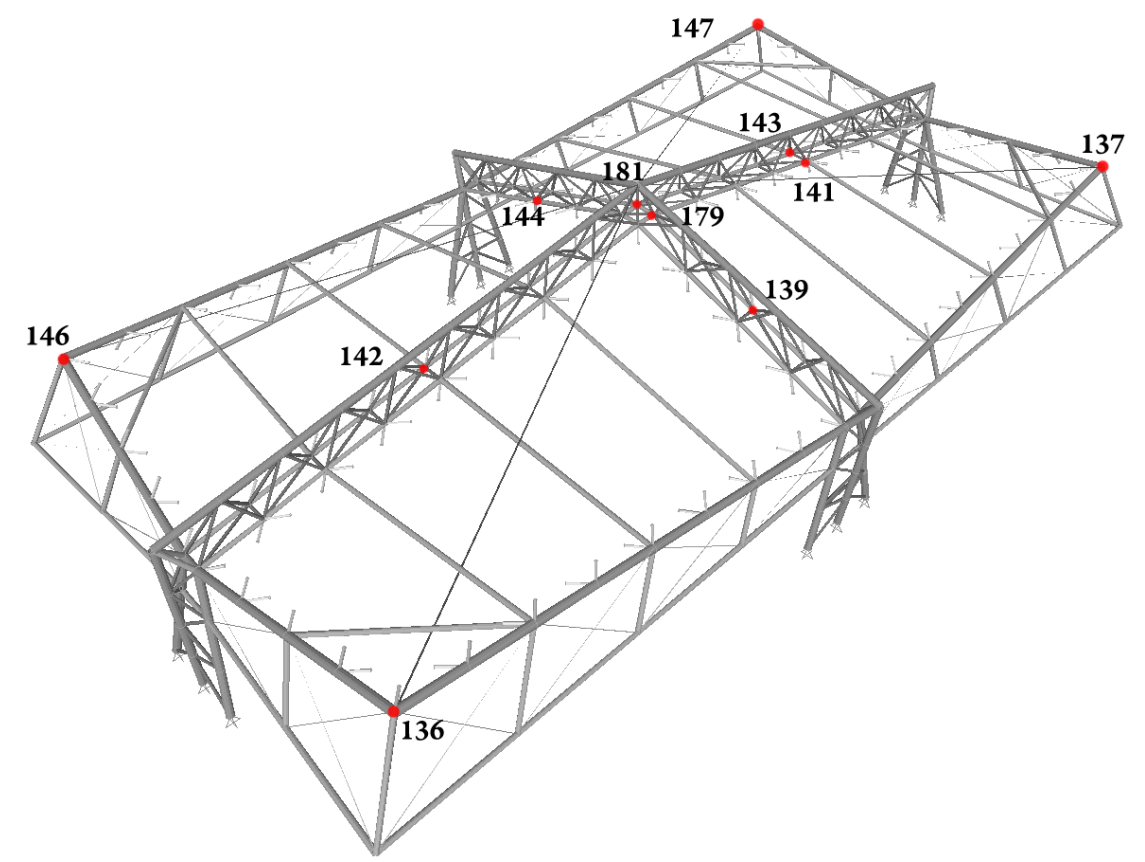

Figura 4.3: OSP obtenida para el método KEMRO 


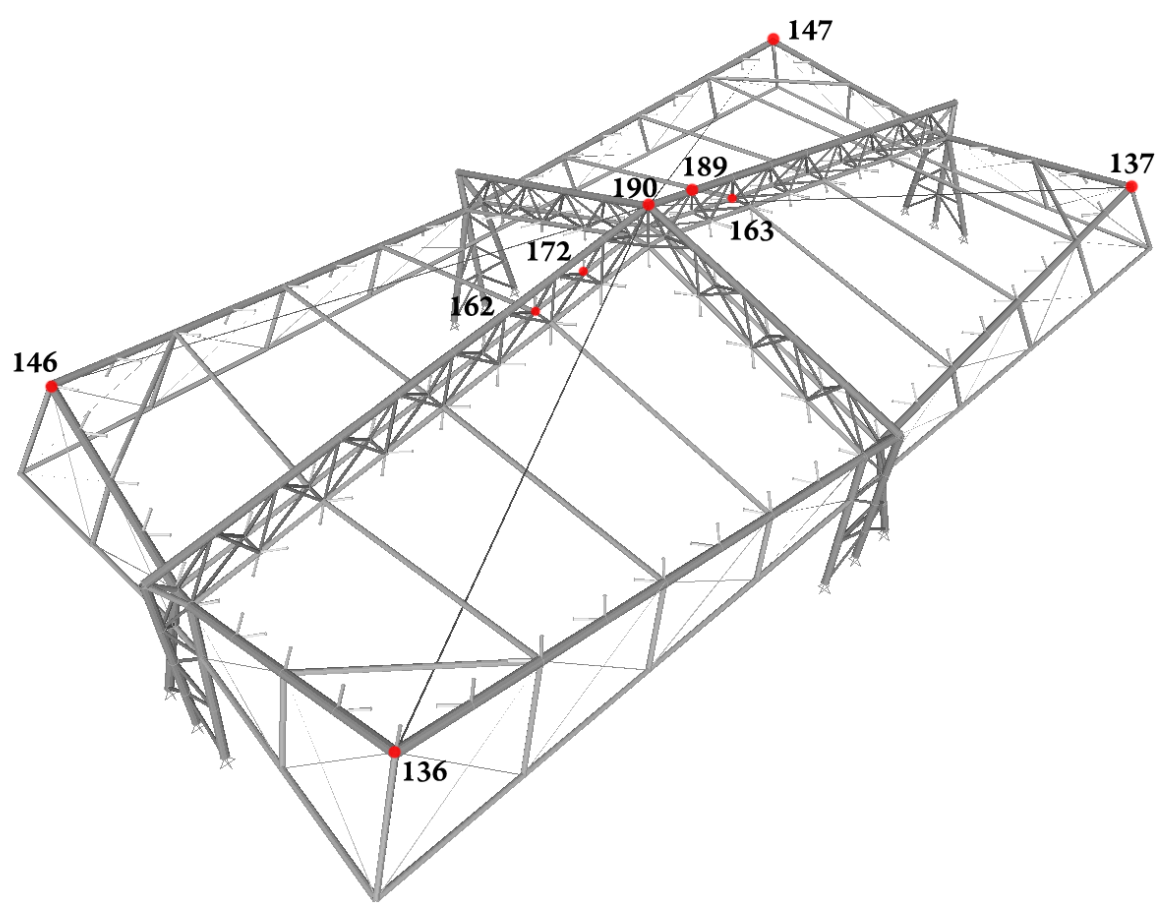

Figura 4.4: OSP obtenida para el método SEMRO

Se observa que aunque los cuatro métodos no tengan los mismos resultados, si presentan una serie lógica.

- Los cuatro métodos seleccionan los cuatro nodos extremos de los voladizos y lo estudian mayoritariamente en el eje Z. El método EFI opta por los nodos que se encuentran en la cara inferior, mientras que los otros métodos se centran en los extremos superiores. En ese punto se concentrarán los mayores desplazamientos y parece una solución lógica del sistema.

- Los cuatro métodos eligen nodos de la cercha superior en sus lados longitudinales, donde la luz es más desfavorable y por tanto se consideran buenos puntos de localización de sensores.

- Se desechan los grados de libertad que se encuentran en los soportes y en puntos próximos a ellos.

\subsubsection{Criterios de evaluación de metodologías OSP}

En la sección anterior, las ubicaciones de los sensores óptimos son elegidos utilizando diferentes metodologías OSP. Por lo tanto, debe establecerse un criterio de evaluación para comparar la idoneidad del sensor con las diferentes configuraciones obtenidas de las 
diferentes metodologías de la OSP. En este apartado se han utilizado tres de los criterios más importantes [2].

1. Determinante de la matriz de información de Fisher (FIM). La definición de la FIM ha sido introducida en la sección 2.1. El factor determinante de la FIM está bien adecuado para la comparación entre las configuraciones de sensores, donde:

$$
\operatorname{det}(\mathbf{F I M})=\operatorname{det}\left(\phi^{T} \phi\right)
$$

Cada vez que una fila es eliminada de la matriz modal $(\phi)$, el determinante de la FIM decrece. El método EFI tiende a mantener el valor con la mayor información retenida. Supongamos el DOF eliminado, entonces si $\phi_{i}$ es la i-ésima fila de $\phi$ :

$$
\begin{gathered}
\mathbf{F I M}=\mathbf{F I M}_{\mathbf{0}}-\phi_{i}^{T} \phi_{i} \\
\mathbf{F I M}=\mathbf{F I M}_{\mathbf{0}}\left(\mathbf{I}-\mathbf{F I M}_{\mathbf{0}}^{-\mathbf{1}} \phi_{i}^{T} \phi_{i}\right)
\end{gathered}
$$

donde $\mathbf{F I M}_{\mathbf{0}}$ corresponde a la matriz del modo $\phi$. Por lo tanto:

$$
\begin{gathered}
\operatorname{det}(\mathbf{F I M})=\operatorname{det}\left(\mathbf{F I M}_{\mathbf{0}}\right) \operatorname{det}\left(\mathbf{I}-\mathbf{F I M}_{\mathbf{0}}^{-\mathbf{1}} \phi_{i}^{T} \phi_{i}\right) \\
\operatorname{det}(\mathbf{F I M})=\operatorname{det}\left(\mathbf{F} \mathbf{I} \mathbf{M}_{\mathbf{0}}\right)\left(1-\phi_{i} \mathbf{F} \mathbf{I M}_{\mathbf{0}}^{-\mathbf{1}} \phi_{i}^{T}\right) \\
\operatorname{det}(\mathbf{F I M})=\operatorname{det}(\mathbf{F I M})_{0}\left(1-\mathbf{P}_{\mathbf{i i}}\right)
\end{gathered}
$$

El término $\mathbf{P}_{\mathrm{ii}}$ representa la contribución del i-ésimo DOF de la matriz FIM. Con cada configuración de sensores hay otros DOFs no considerados, por lo tanto el determinante de la Matriz de Fisher disminuye. A la hora de evaluar dos diferentes configuraciones de sensores, un criterio adecuado seria comparar dicho valor.

2. Criterio de adecuación modal (MAC). Originalmente este criterio fue usado para asegurar la correlación entre las formas modales de un modelo numérico $\left(\phi_{\alpha}\right)$ y las formas modales de un examen experimental $\left(\phi_{e}\right)$, por ejemplo en análisis modal experimental u operacional. Entonces los términos $(i, j)$ de la matriz MAC son definidos como:

$$
M A C_{i j}=\frac{\left(\phi_{\alpha i}^{T} \phi_{e j}\right)^{2}}{\left(\phi_{\alpha i}^{T} \phi_{\alpha j}\right)\left(\phi_{e i}^{T} \phi_{e j}\right)}
$$

Si un término de la $M A C_{i j}$ es cero, entonces los vectores de $\phi_{\alpha i} \mathrm{y} \phi_{e j}$ no estarán correlados. Si la $M A C_{i j}$ es 1 entonces los modos están completamente correlados. En el caso del presente artículo sólo las formas de los modos analíticos se comparan, y sólo en los sensores donde los DOFs se conservan. Como consecuencia de los términos de la diagonal de la MAC siempre son iguales a 1, aunque los términos de fuera de la diagonal serán entre 0 y 1 . Así la mejor configuración de sensores 
es la que tiene terminos bajos fuera de la diagonal. Para obtener una medida de la calidad de una configuración de sensores, la raíz media cuadrática (RMS) de los términos de fuera de la diagonal MAC es calculada como:

$$
\sqrt{\frac{1}{N(N-1)} \sum_{i=1}^{N} \sum_{j=1 \neq i}^{N}\left(M A C_{i j}\right)^{2}}
$$

tendiendo en cuenta que $i=1, \ldots, N$ y también $j=1, \ldots, N$.

Un buen procedimiento para probar la exactitud de la configuración del sensor seleccionado es comparar el valor RMS de los términos de fuera de la diagonal con el caso donde son considerados todos los DOFs.

3. Singular value decomposition ratio (SVD-ratio). En este caso, la matriz de formas modales es descompuesta en el producto de tres matrices diferentes, $\mathrm{U}, \Sigma$ y V, de la siguiente forma:

$$
\phi_{m \times n}=\mathbf{U}_{m \times n} \boldsymbol{\Sigma}_{n \times n} \mathbf{V}_{n \times n}^{T}
$$

La matriz $\Sigma$ es diagonal donde los términos son conocidos como valores singulares de la matriz de modos $\phi$. El número de términos de la diagonal distinto de cero determinan el rango de la matriz descompuesta. Las otras dos matrices, $\mathrm{U}$ y V, son matrices ortogonales. Si las formas modales son ortogonales, todos los términos de la matriz modal son iguales. Si los vectores son linealmente dependientes, al menos un valor singular será cero. Para comparar las distintas cofiguraciones de sensores, se usa el ratio entre los valores más alto y el más bajo de los valores singulares de descomposición de la matriz calculada de formas modales . Cuando los ratios salen muy altos, siginficará que la configuración de sensores elegidas es muy pobre.

El cuadro 4.3 presenta los valores obtenidos para las cuatro metodologías aplicadas en el caso determinista. Se ha marcado las mejores soluciones para cada criterio de calidad.

\begin{tabular}{lccc}
\hline Método & $\mu(\operatorname{det}(\mathrm{FIM}))$ & $\mu$ (MAC rms error) $)$ & $\mu$ (SVD-ratio)) \\
\hline EFI & $\mathbf{0 . 3 2 0 6}$ & 0.1113 & 3.6080 \\
EFIWM & 0.0012 & 0.1031 & 7.2712 \\
KEMRO & 0.0029 & $\mathbf{0 . 0 9 9 3}$ & $\mathbf{3 . 2 2 9 2}$ \\
SEMRO & $4.9738 \times 10^{-5}$ & 0.1549 & 7.9463 \\
\hline
\end{tabular}

Cuadro 4.3: Criterios de calidad de la medición de OSP

Se observa que la mejor solución en el caso de la maximización del determinante de FIM es el método EFI, al ser elegido como valor máximo. En cambio, para los otros criterios de calidad, la metodología KEMRO es la mejor opción. El algoritmo de KEMRO es el que nos ofrece una solución más pobre en la evaluación de los grados de libertad. 


\subsection{Simulación de Monte Carlo (MCS).}

Los análisis de incertidumbre puede realizarse con varios métodos estocásticos disponibles en la literatura [31] La Simulación de Monte Carlo (denominada con las iniciales MCS del inglés Monte Carlo Simulation) es la tecnica de análisis más precisa [32, 34, 35]. La principal ventaja de MCS es que pueden realizarse análisis de incertidumbre sin ninguna modificación del programa de análisis de elementos finitos. El MCS requiere un gran número de simulaciones y resultados con un alto coste computacional. Aunque el modelo elegido complejice la tarea, la aplicación desarrollada nos produce miles de cálculos iterativos sin apenas esfuerzo.

El análisis de incertidumbre se puede definir según los siguientes apartados:

- Selección de los parámetros bajo incertidumbre. Los parámetros correspondientes a la geometría y a las propiedades del material de las barras pueden ser variables a causa de la tolerancias de error y tratamientos térmicos durante su fabricación, o variaciones en la temperatura ambiente. En el este trabajo, las propiedades del material y la sección de las barras son consideradas como variables aleatorias con distribución gaussiana. Los valores promedios y coeficientes de variación $(\mathrm{CoV})$ de estos parámetros que se han tomado son los correspondientes a una publicación con un estudio previo ya existente [44], que en nuestro caso son los siguientes:

\begin{tabular}{lccc}
\hline Elemento & Media & CoV & Distribución \\
\hline P. Tubular1, mm & 323 & $2 \%$ & Normal \\
P. Tubular2, mm & 200 & $2 \%$ & Normal \\
P. Tubular3, mm & 120 & $2 \%$ & Normal \\
Módulo de Elasticidad, GPa & 200 & $8 \%$ & Normal \\
Densidad del acero, $\mathrm{Kg} / \mathrm{m}^{3}$ & 7850 & $4 \%$ & Normal \\
\hline
\end{tabular}

Cuadro 4.4: Las propiedades estocásticas de los parámetros de la estructura.

- Generación de muestras de parámetros estocásticos. Un muestreo esquemático aleatorio usando MATLAB se utiliza para generar las muestras aleatorias de los parámetros elegidos. El Módulo de Young $\left(E_{1}\right)$, densidad $\left(\rho_{1}\right)$ y las secciones de los perfiles tubulares $(A)$ son generadas y consideradas variables aleatorias independientes. La Simulación de Monte Carlo se realiza con el código de elementos finitos determinista para el número de muestras. Para este trabajo se han considerado dos mil muestras, ya que en estudios previos se ha estudiado que a ese nivel las medias de los parámetros llegan a tener un valor estable. 
Capítulo 4. Resultados.

\subsection{Localización de sensores bajo incertidumbre paramétrica}

\subsubsection{Efectos de las incertidumbres sobre la OSP}

Inicialmente, el efecto de parámetros inciertos sobre la configuración del sensor es estudiado independientemente.11 localizaciones de sensores son escogidos de entre los 570 DOFs disponibles utilizando los cuatro Métodos OSP descritos en la sección 3. En MCS, las propiedades geométricas y transversales varían con cada muestra. Por lo tanto, también varía la ubicación óptima de los sensores en cada una de las muestras. Las probabilidades de los DOFs elegidos como ubicaciones de los sensores óptimos, para 2000 iteraciones se muestran en 4.5 ,

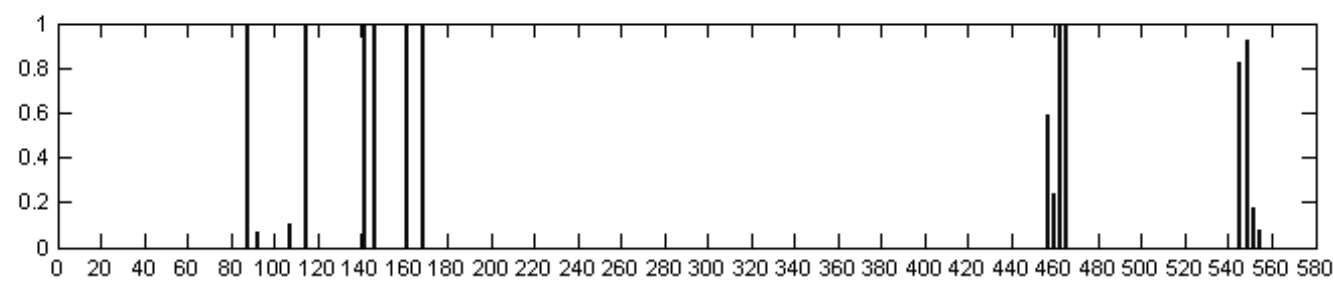

(a) Solución de EFI con incertidumbre en el módulo de Young

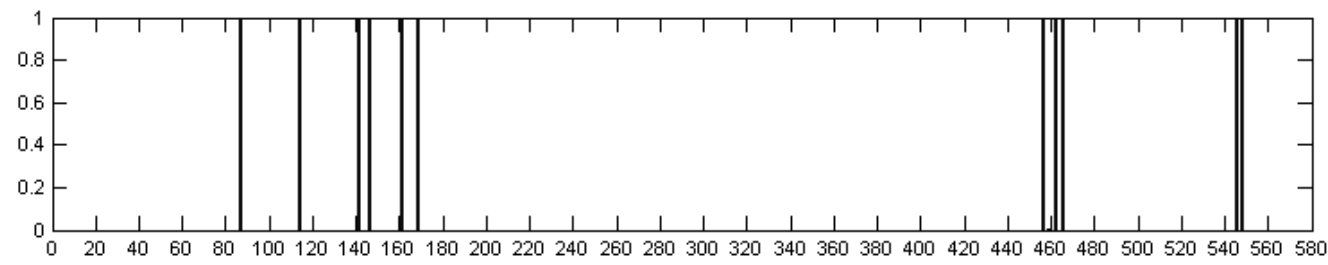

(b) Solución de EFI con incertidumbre en la densidad del material

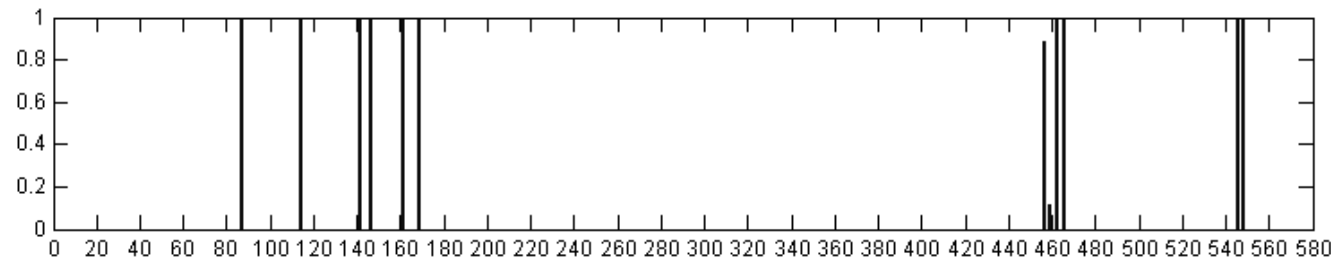

(c) Solución de EFI con incertidumbre en los perfiles

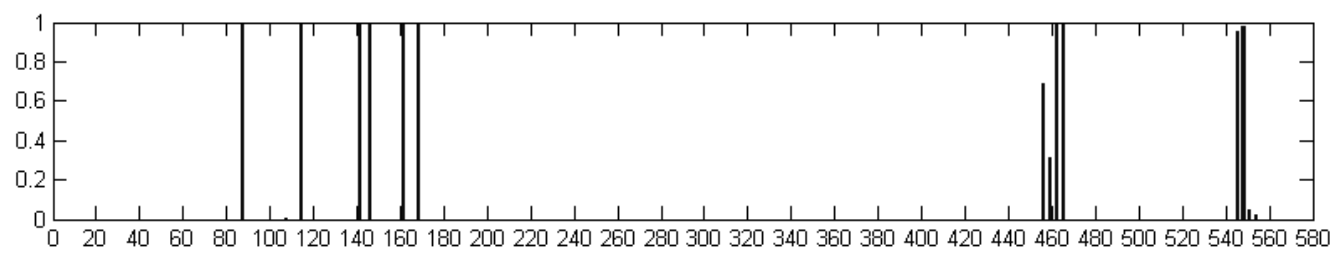

(d) Solución de EFI con todas las incertidumbres

Figura 4.5: Distintas soluciones tomando distintas incertidumbres 
Los resultados muestran que hay varios DOFs que siempre son elegidos como una ubicación del sensor óptimo en cada muestra de la MCS. Estas ubicaciones de los sensores son de vital importancia en la configuración global y se puede llamar como sensores vitales. El resultados de diferentes métodos OSP aparecen a lo largo de las diferentes filas y el efecto de cada parámetro incierto se presenta en diferentes columnas. Los resultados son contundentes, con la aplicación de incertidumbres en el método EFI prácticamente salen 10 grados de libertad vitales de los 11 buscados. Estos misma localización se compara con el resultado determinista, que coincide y sugiere el mismo resultado.

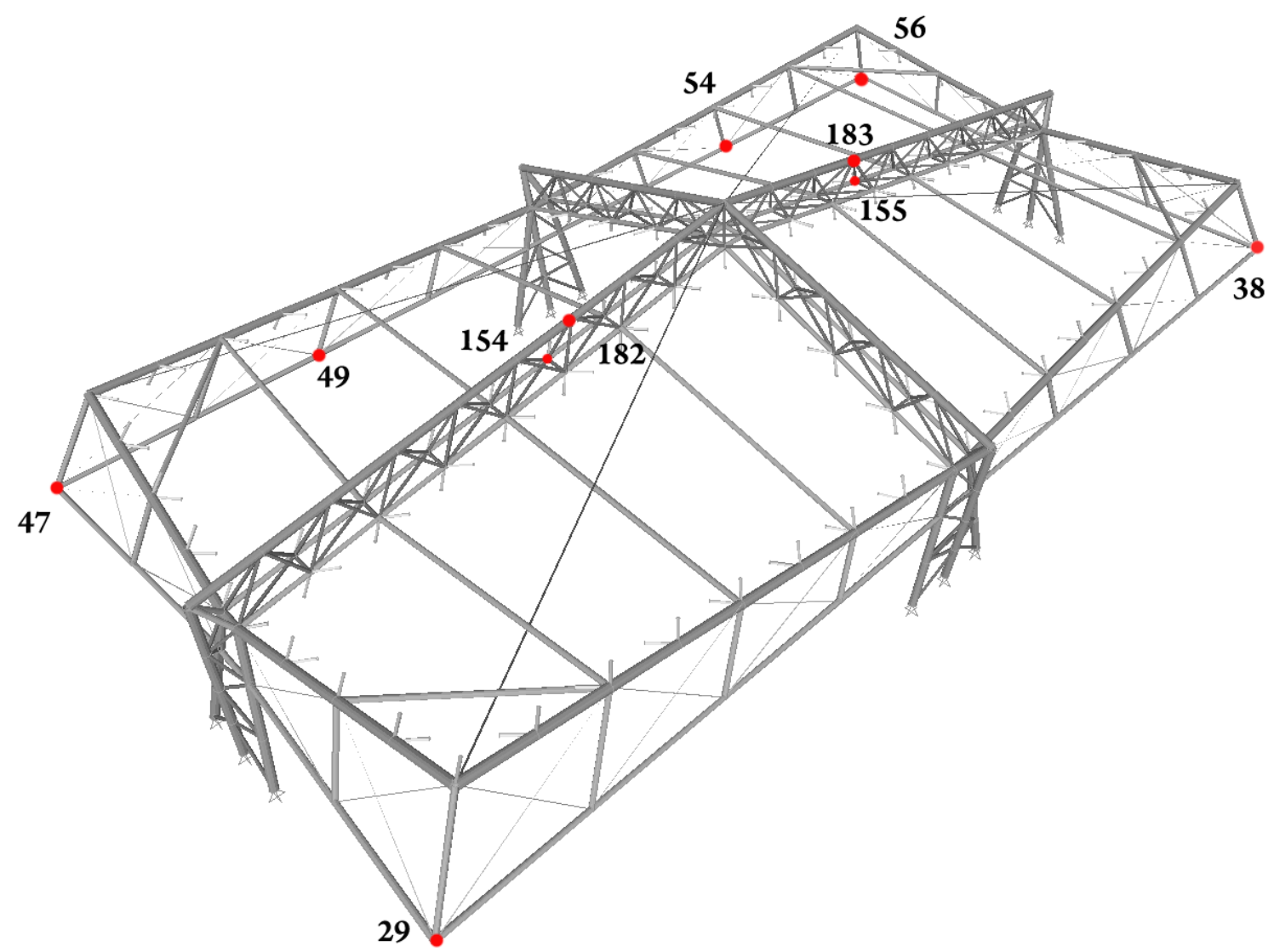

Figura 4.6: OSP obtenida para el método EFI

\subsubsection{Criterios de evaluación de metodologías OSP}

Como se ha descrito en el cálculo determinista, debe establecerse un criterio de evaluación para comparar la idoneidad del sensor con las diferentes configuraciones obtenidas de las diferentes metodologías de la OSP y las diferentes incertidumbres. En este apartado se presenta el resultado de las distintas evoluciones de los criterios por probabilidad de ocurrencia. Estos tres criterios de calidad se han aplicado a cada muestra que 
hemos obtenido de Montecarlo, aplicando en este caso el método EFI. Se han ploteado histogramas donde se representa la distribución de los distintos criterios de calidad para distintas distribuciones de Montecarlo.

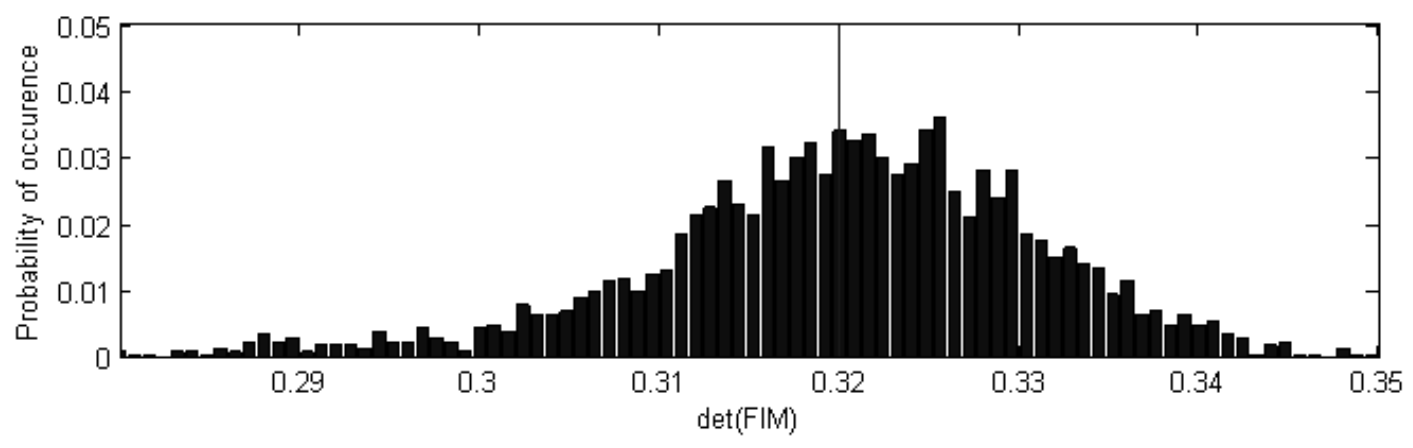

(a) Evolución del $\operatorname{det}(\mathrm{FIM})$ con EFI aplicado a todas las incertidumbres paramétricas

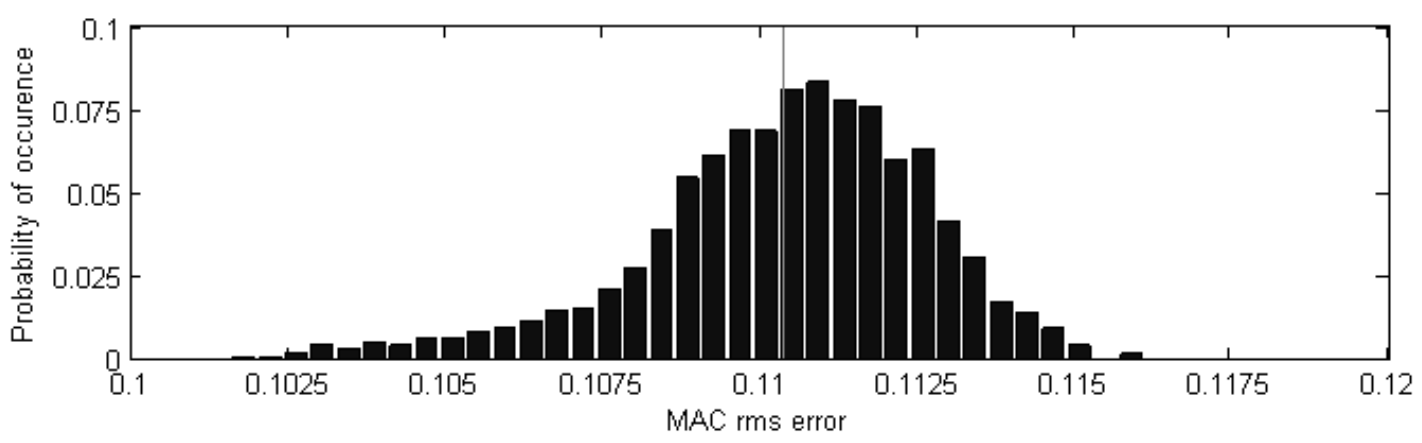

(b) Evolución de MAC rms error con EFI aplicado a todas las incertidumbres paramétricas

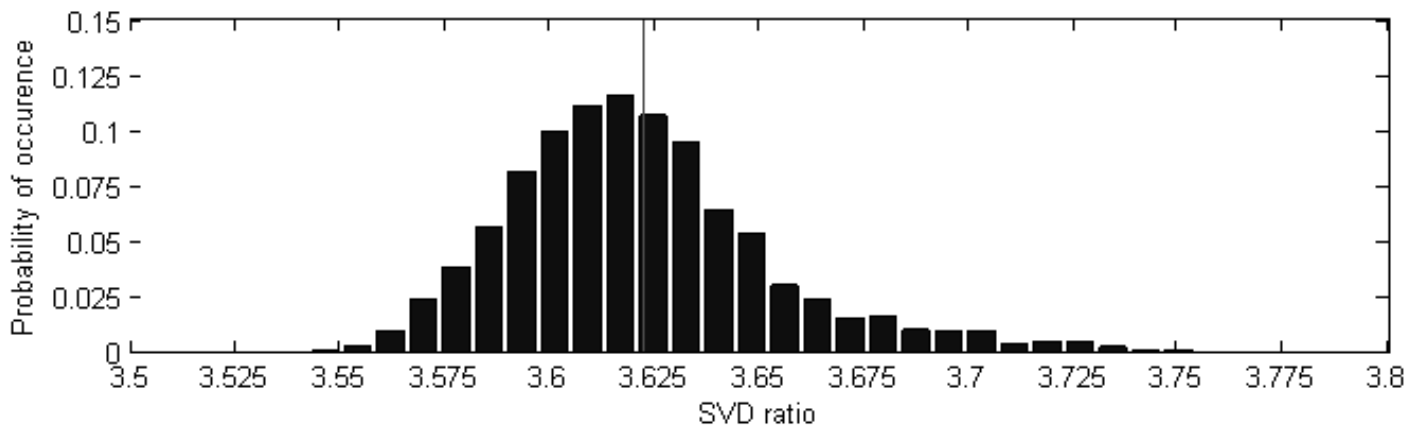

(c) Evolución de SVD ratio con EFI aplicado a todas las incertidumbres paramétricas

Figura 4.7: Histogramas de los criterios de evaluación. 


\subsection{Configuración óptima de sensores con ruido.}

Las técnicas de medición de análisis modal operacional y experimental están sujetas a efectos de ruido que contaminan los datos experimentales. La configuración de sensores debe contar con la existencia de éstos. De esa manera, se puede determinar el número de sensores necesarios que deben ser empleados en las pruebas experimentales. Por lo tanto, un cierto nivel de certeza es necesaria para demostrar la calidad de la conguración de sensores.

Los efectos de ruido son incorporados numéricamente al modelo de elementos finitos para simular las consecuencias. La relación señal/ruido, más conocido por su nombre en inglés signal-to-noise ratio (SNR) es empleado para la medición del nivel de ruido. El SNR es definido como el ratio potencial entre la señal (información significativa) y el ruido de fondo (información no deseada).

$$
S N R=\frac{P_{\text {senal }}}{P_{\text {ruido }}}
$$

En este caso el ruido es añadido a la información significativa de los objetivos obtenidos en la OSP, añadido a las formas modales. Han sido considerados diferentes niveles de $\mathrm{SNR}$, desde el caso de un ruido muy leve $(\mathrm{SNR}=40 \mathrm{~dB})$, un nivel medio $(\mathrm{SNR}=30 \mathrm{~dB}$ ) $y$ en el caso de un nivel de ruido bastante alto $(S N R=10 \mathrm{~dB})$.

En el caso ideal de que no haya ruido, la evolución del MAC rms error para diferentes configuraciones de sensores refleja cómo este valor mejora cuando se incrementa el número de sensores. Se han ejecutado diversas rutinas de ruido con distintos números de sensores, y se puede ver en el cuadro 4.5 que existe una tendencia clara y esperada, cuando hay un mayor número de sensores, el error se reduce, como se describe. En cambio, con ruido presente, el error MAC incrementa. Igualmente, cuando tenemos un número de sensores menor, los resultados son altos al considerarse una localización de sensores pobre.

\begin{tabular}{lcccc}
\hline & \multicolumn{4}{c}{$\mu$ (MAC rms error) $)$} \\
& sin ruido & $40 \mathrm{~dB}$ & $30 \mathrm{~dB}$ & $10 \mathrm{~dB}$ \\
\hline 8 sensores & 0.1570 & 0.1571 & 0.1614 & 0.1733 \\
11 sensores & 0.1113 & 0.1115 & 0.1132 & 0.1241 \\
14 sensores & 0.1001 & 0.1017 & 0.1044 & 0.1125 \\
\hline
\end{tabular}

Cuadro 4.5: Relación del MAC rms error para distinto número de sensores y distintos valores de ruido

Viendo esta posibilidad, es posible obtener el número de sensores deseados si se busca una nivel de aproximación específico. Si hay que tener en cuenta que cuántos más sensores sean necesarios en la configuración, más se encarecerá, llegando incluso a resultar determinante. La respuesta no siempre puede ser cuantos más sensores mejor, ya 
que puede ser que entre un número de sensores y otro, aplicando el ruido, no haya tanta diferencia y podría economizar la configuración.

En los histogramas de la figura 4.8 se puede ver la tendencia que obtiene el error a distintos niveles de ruido. Se observa que a un ratio bajo el MAC rms error arroja valores considerablemente altos, si se compara con los histogramas sin ruido. 


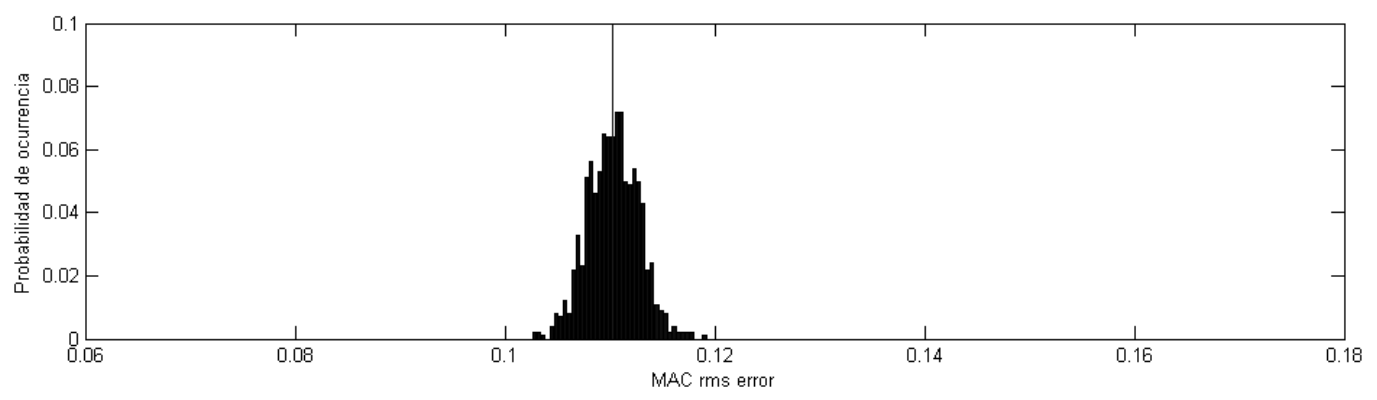

(a) Evolución de MAC rms error sin ruido

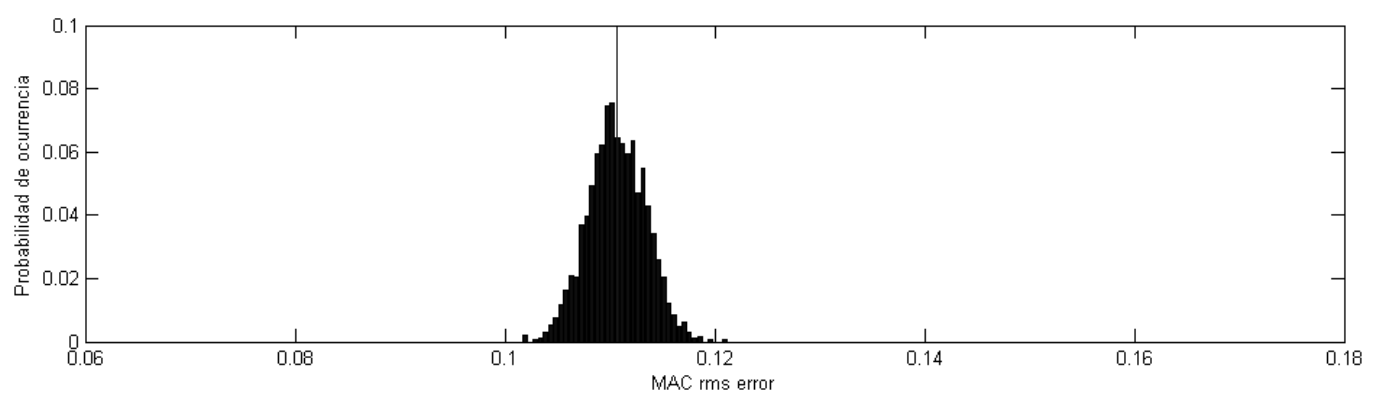

(b) Evolución de MAC rms error con $40 \mathrm{~dB}$

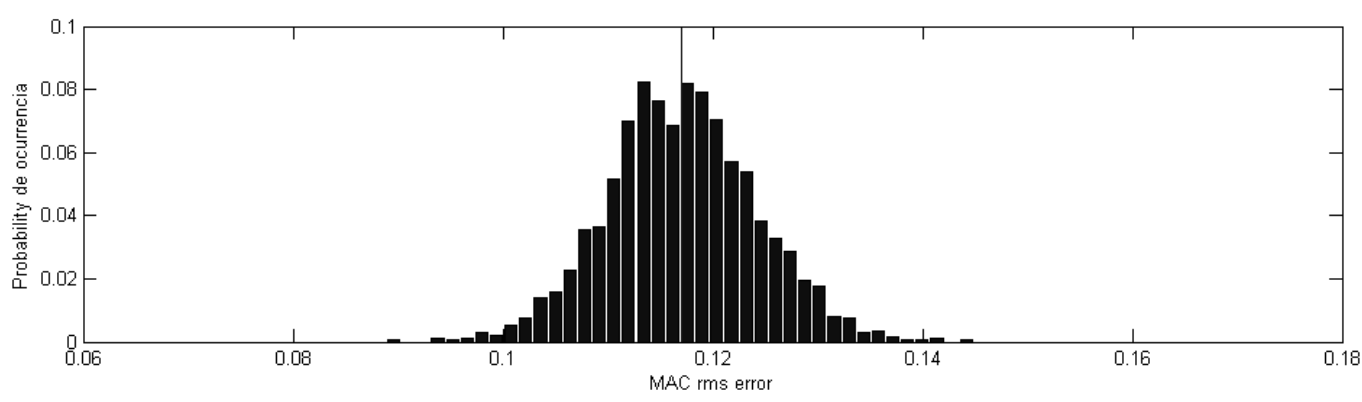

(c) Evolución de MAC rms error con $30 \mathrm{~dB}$

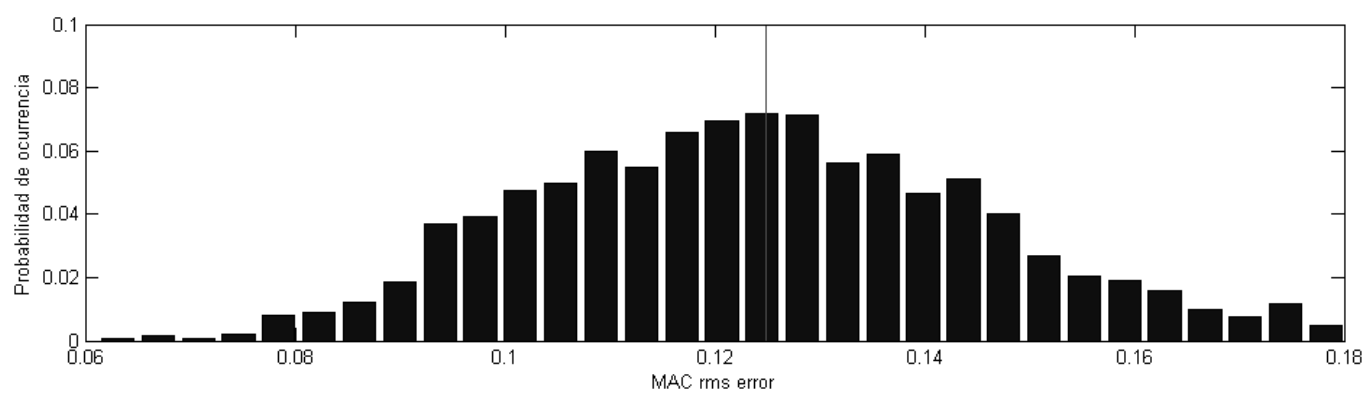

(d) Evolución de MAC rms error con $10 \mathrm{~dB}$

Figura 4.8: Histogramas del criterio de evaluación aplicando el MAC rms error para distintos niveles de ruido con 11 sensores. 
Capítulo 4. Resultados. 


\section{Capítulo 5}

\section{Conclusiones y Trabajo Futuro.}

En este capítulo se explican las conclusiones finales de este trabajo, y se comentarán algunas líneas de trabajo futuro.

\subsection{Conclusiones.}

En este trabajo se ha presentado la aplicación de distintas metodologías de Análisis Modal Operacional para la localización óptima de sensores. Estas metodologías se han aplicado iterativamente para una serie de casos, introduciendo incertidumbres que puedan dar resultados de sensores vitales en el emplazamiento óptimo.

Se presentan cuatro métodos para un cálculo de la estructura sin incertidumbres. Se han obtenido cuatro localizaciones de sensores distintas, que tienen algunos grados de libertad en común o siguen un esquema lógico. A estas localizaciones se les ha aplicado tres criterios de evaluación de demuestran la calidad de la localización obtenida, estableciendo una tabla resumen.

Se introducen incertidumbres paramétricas al modelo a través de un algoritmo de Matlab, de forma externa al programa de análisis de elementos finitos. En este caso se han introducido bajo incertidumbre la densidad del material, el módulo de Young y la sección de las barras. Tras iterativos cálculos para la metodología EFI, en los que las incertidumbres se ejecutaban individual y conjuntamente, se obtienen una localizaciones de sensores OSP. Dichas localizaciones demuestran que hay poca variabilidad y que afianzan una serie de grados de libertad, que se denominan vitales.

A estos cálculos iterativos también se les introduce unos criterios de evaluación, de los que se extrae una media muy similar al cálculo determinista.

Como último punto de la memoria, se introduce a los cálculos distintos niveles de ruido, que muestran a través del criterio de evaluación MAC rms error que incrementa 
su evaluación al nivel de $10 \mathrm{~dB}$, mientras que apenas hay diferencias entre una $\mathrm{SNR}=40 \mathrm{~dB}$ y una evolución sin ruido.

Este trabajo fin de máster es un punto de partida a el cálculo de incertidumbres y análisis modal operacional de estructuras complejas. La capacidad de cálculo no había permitido hasta ahora realizar miles de iteraciones sin que tuvieran que significar varios días y sumándole la posibilidad de ejecutarse iterativamente de manera automática. Es por ello que este trabajo es importante para la comunidad del análisis modal.

\subsection{Trabajo futuro.}

En esta sección, vamos a distinguir algunas líneas de trabajo futuro, divididas en dos ámbitos de aplicación: Investigación y Aplicación comercial.

En este apartado, vamos a describir algunas líneas de investigación. Podemos enumerar los siguientes puntos:

- Este trabajo ha detallado las incertidumbres paramétricas con el método EFI. Se podrían estudiar otras metodologías, también descritas en este trabajo, como son EFIWM, KEMRO y SEMRO.

- Se ha demostrado que es posible realizar cálculos iterativos de estructuras 3D complejas. Hoy en dia los ordenadores son cada vez más veloces y nos permiten realizar una iteración de una estructura compleja en 30 segundos.

- Para este trabajo se ha creado una herramienta que posibilita que los datos de un modelo de elementos finitos en SAP2000 pueda ser .exportadoz trabajado desde Matlab. Dicha herramientra podría trabajar con cualquier modelo, posibilitando distintos trabajos futuros.

- La incorporación a este trabajo de un estudio experimental de la estructura podria corroborar los análisis teóricos mostrados. 


\section{Bibliografía}

[1] C. R. Pickrel. A practical approach to modal pretest design. Mechanical Systems and Signal Processing, 13:271-295, (1999).

[2] J. E. T. Penny, M. I. Friswell, and S. D. Garvey. Automatic choice of measurement locations for dynamic testing. AIAA Journal, 32:407-414, (1994).

[3] M. Papadopoulos and E. Garcia. Sensor placement methodologies for dynamic testing. AIAA Journal, 36:256-263, (1998).

[4] D. S. Li and H. N. Li. The state of the art of sensor placement methods in structural health monitoring. Smart Structures and Materials 2006: Sensors and Smart Structures Technologies for Civil, Mechanical, and Aerospace Systems, 6174:1217-1227, (2006).

[5] D. C. Kammer. Sensor placement for on-orbit modal identification and correlation of large space structures. Journal of Guidance, Control and Dynamics, 14:251-259, (1991).

[6] D. C. Kammer and M. L. Tinker. Optimal placement of triaxial accelerometers for modal vibration tests. Mechanical Systems and Signal Processing, 18:29-41, (2004).

[7] D. C. Kammer and J. A. Peck. Mass-weighting methods for sensor placement using sensor set expansion techniques. Mechanical Systems and Signal Processing, 22:1515-1525, (2008).

[8] F. M. Hemez and C. Farhat. An energy based optimum sensor placement criterion and its application to structural damage detection. XII International Modal Analysis Conference (IMAC), Ilikai Hotel, Honolulu, Hawaii:1568-1575, 1994.

[9] G. Heo, M. L. Wang, and D. Satpathi. Optimal transducer placement for health monitoring of long span bridge. Soil Dynamics and Earthquake Engineering, 16:495-502, (1997).

[10] D. C. Kammer and L. Yao. Enhancement of on-orbit modal identification of large space structures through sensor placement. Journal of Sound and Vibration, 171:119-139, (1994).

[11] R. S. Glassburn and S. W. Smith. Evaluation of sensor placement algorithms for on-orbit identification of space platforms. NASA XVIII Space Simulation Conference: Space Mission Sucess through Testing, page 333-348, (1994). 
[12] F. Udwadia. Methodology for optimum sensor locations for parameter identification in dynamic systems. Journal of Engineering Mechanics, 120:368-390, (1994).

[13] P. H. Kirkegaard and R. Brincker. On the optimal location of sensors for parametric identification of linear structural systems. Mechanical Systems and Signal Processing, 8:639-647, (1994).

[14] D. C. Kammer. Optimal sensor placement for modal identification using systemrealization methods. Journal of Guidance, Control and Dynamics, 19:729-731, (1996).

[15] C. Papadimitriou. Optimal sensor placement methodology for parametric identification of structural systems. Journal of Sound and Vibration, 278:923-947, (2004).

[16] S. J. Kwon. Determination of optimal accelerometer locations using modal sensitivity for identifying a structure. Smart Structures and Systems, 5:629-640, (2008).

[17] C. Stephan. Sensor placement for modal identification. Mechanical Systems and Signal Processing.

[18] Y. J. Yan, L. Cheng, Z. Y.Wu, and L. H. Yam. Development in vibration-based structural damage detection technique. Mechanical Systems and Signal Processing, 21:2198-2211, (2007).

[19] P. S. Skjaerbaek and R. K. Nielsen. Identification of damage in reinforced concrete structures from earthquake records - optimal location of sensors. Soil Dynamics and Earthquake Engineering, 15:347-358, (1996).

[20] K. Worden and A. P. Burrows. Optimal sensor placement for fault detection. Engineering Structures, 23:885-901, (2001).

[21] P. Kripakaran and I. F. C. Smith. Configuring and enhancing measurement systems for damage identification. Advanced Engineering Informatics, 23:424-432, (2009).

[22] S. K. Hong, B. I. Epureanu, and M. P. Castenier. Novel sensor placement for damage identification in a cracked complex structure with structural variability. Journal of Intelligent Material Systems and Structures, 22:1189-1202, (2011).

[23] O. A. Vanli, C. Zhang, A. Nguyen, and B. Wang. A minimax sensor placement approach for damage detection in composite structures. Journal of Intelligent Material Systems and Structures, 23:919-932, (2012).

[24] A. M. Raich and T. R. Liszkai. Multi-objective optimization of sensor and excitation layouts for frequency response function-based structural damage identification. Computer-Aided Civil and Infrastructure Engineering, 27:95-117, (2012).

[25] Z. Y. Shi, S. S. Law, and L. M. Zhang. Optimum sensor placement for structural damage detection. Journal of Engineering Mechanics, 126:1173-1179, (2000).

[26] H. Y. Guo, L. Zhang, L. L. Zhang, and J. X. Zhou. Optimal placement of sensors for structural health monitoring using improved genetic algorithms. Smart Materials and Structures, 13:528-534, (2004). 
[27] M. I. Friswell and J. E. Mottershead. Finite element model updating in structural dynamics. Kluwer Academic Publishers, 1995.

[28] M. Reynier and H. A. Kandil. Sensors location for updating problems. Mechanical Systems and Signal Processing, 13:297-314, (1999).

[29] C. Papadimitriou and J. L. Beck. Entropy-based optimal sensor location for structural model updating. Journal of Vibration and Control, 6:781-800, (2000).

[30] H. F. Lam, J. H. Yang, and Q. Hu. How to install sensors for structural model updating? Procedia Engineering, 14:450-459, (2011).

[31] G. I. Schueller. On the treatment of uncertainties in structural mechanics and analysis. Computers and Structures, 85:235-243, (2007).

[32] S. K. Choi, R. V. Grandhi, and R. A. Canfield. Reliability-based structural design. Springer, (2006).

[33] S. W. Doebling and F. M. Hemez. Overview of uncertainty assessment for structural health monitoring. Proceedings of the 3rd International Workshop on Structural Health Monitoring, Stanford University, Stanford, California, (2001).

[34] S. Murugan, R. Ganguli, and D. Harursampath. Aeroelastic response of composite helicopter rotor with random material properties. Journal of aircraft, 45:306-322, (2008).

[35] S. Murugan, D. Harursampath, and R. Ganguli. Material uncertainty propagation in helicopter nonlinear aeroelastic response and vibration analysis. AIAA journal, 46:2332-2344, (2008).

[36] S. Murugan, R. Chowdhury, S. Adhikari, and M. I. Friswell. Helicopter aeroelastic analysis with spatially uncertain rotor blade properties. Aerospace Science and Technology, 16:29-39, (2011).

[37] M. Azarbayejani, A. I. El-Osery, K. K. Choi, and M. M. R. Taha. A probabilistic approach for optimal sensor allocation in structural health monitoring. Smart Materials and Structures 17, pages 1-11, (2008).

[38] F. Escrig, J. Sánchez, and M. T. Rodríguez León. Cubierta para un recinto multifuncional abierto con cuatro paraboloides hiperbolicos textiles, (2012).

[39] L. Álvarez, J. R. Barroso, M. Galindo, S. Girón, and T. Zamarreño. Acústica de una pista polideportiva multiusos con cubierta en membrana textil tensionada. Congreso Español de Acústica European Symposium on Environmental Acoustics and on Building Acoustically Sustainable, TecniAcústica. Cáceres, (2011).

[40] S. D. Garvey, M. I. Friswell, and J. E. T. Penny. Evaluation of a method for automatic selection of measurement locations based on subspace-matching,. XIV International Modal Analysis Conference (IMAC), Hyatt Regency Dearborn Hotel, Dearborn, Michigan:1546-1552, (1996). 
[41] J. A. Lollock and T. R. Cole. The effect of mass-weighting on the effective independence of mode shapes. 46th AIAA/ASME/ASCE/AHS/ASC Structures, Structural Dynamics and Materials Conference, Austin, Texas, USA:1-8, (2005).

[42] M. Meo and G. Zumpano. On the optimal sensor placement techniques for a bridge structure. Engineering Structures, 27:1488-1497, (2005).

[43] M. Meo and G. Zumpano. Optimal sensor placement on large scale civil structure. Health Monitoring and Smart Nondestructive Evaluation of Structural and Biological Systems III, (2004).

[44] R. F. Guratzsch and S. Mahadevan. Structural health monitoring sensor placement optimization under uncertainty. AIAA Journal, 48:1281-1289, (2010). 